\title{
Title
}

\section{Identification of QPCR reference genes suitable for normalising gene expression in the developing mouse embryo}

\author{
John C.W. Hildyard ${ }^{1 *}$, Dominic J. Wells ${ }^{2}$, Richard J. Piercy ${ }^{1}$ \\ ${ }^{1}$ Comparative Neuromuscular Diseases Laboratory, Department of Clinical Science and \\ Services, Royal Veterinary College, London NW1 0TU, United Kingdom \\ ${ }^{2}$ Department of Comparative Biomedical Sciences, Royal Veterinary College, London NW1 \\ OTU, United Kingdom \\ * Corresponding author: jhildyard@rvc.ac.uk
}

\begin{abstract}
Mammalian embryogenesis is an intricate, tightly orchestrated process. Progression from zygote through somitogenesis and on to organogenesis and maturity involves many interacting cell types and multiple differentiating cell lineages. Quantitative PCR analysis of gene expression in the developing embryo is a valuable tool for deciphering these interactions and tracing lineages, but normalisation of qPCR data to stably expressed reference genes is essential. Patterns of gene expression change globally and dramatically as embryonic development proceeds, rendering identification of appropriate reference genes challenging at both the whole embryo- and individual tissue-level. We have investigated expression stability in mouse embryos from mid to late gestation (E11.5-E18.5), both at the whole-embryo level, and within more restricted tissue domains (head, developing forelimb), using 15 candidate reference genes (ACTB, 18S, SDHA, GAPDH, HTATSF1, CDC40, RPL13A, CSNK2A2, AP3D1, HPRT1, CYC1, EIF4A, UBC, B2M and PAK1IP1), and four complementary algorithms (geNorm, Normfinder, Bestkeeper and deltaCt). Unexpectedly, all methods suggest that many genes within our candidate panel are acceptable references, and despite disagreement over highest-scoring candidates, AP3D1, RPL14A and PAK1IP1 are the strongest performing genes overall. Conversely, HPRT1 and B2M are consistently poor choices: these genes show strong developmental regulation. We further show that use of $A P 3 D 1, R P L 13 A$ and PAK1IP1 can reveal subtle patterns of developmental expression even in genes ostensibly ranked as acceptable (CDC40, HTATSF1), and thus these three represent universally suitable reference genes for the mouse embryo.
\end{abstract}

\section{Introduction}

The mouse (Mus musculus) is the principal model organism for the study of mammalian embryonic development, offering large litter sizes and a predictable gestation period (mating in this species can moreover be timed with relative ease). The stages of mouse embryonic 
development are thus well documented [1, 2], and given the conserved nature of mammalian embryogenesis, can also be mapped across mammalian species despite gestation times differing by more than an order of magnitude (20 days in the mouse, 270 days in the human, up to 645 days in the African elephant Loxodonta) [3].

The earliest stages of development establish fundamental morphological patterns: blastocyst formation, implantation, establishment of polarity occur with the first five days (Embryonic days 1-5, or E1-E5) [4], with gastrulation and development of the primitive streak following (E6-E8.5) [5]. Intermediate stages (E8.5-E10.5) feature the cyclical 'clock and wave' of somitogenesis [6], the turning of the embryo, neural tube closure and the laying down of organ precursor cell lineages, while the later stages (E11.5 onward) involve maturation and development of those organs [1, 2]. During this later period (from E11.5 to E18.5, Fig 1) almost all limb development occurs, from a primitive limb bud to an essentially mature state complete with ossifying bones and fully-defined joints and digits [7-9], and similarly almost all skeletal muscle is laid down (both primary and secondary myogenesis) [10,11]. The head undergoes a broad panel of changes, with the brain enlarging and maturing [12], the palate closing and teeth forming [13], the ears emerging, and during this period the eye progresses from a simple indentation to a fully encapsulated globe (which is subsequently sheltered beneath fused eyelids) [14]. More globally, this later period also spans substantial changes in haematopoiesis (with the source of blood cells switching from the yolk sac to the liver, and then subsequently to the bone marrow) [15], maturation of the chambers of the heart [16], gradual replacement of the mesonephros with the metanephros [17], and establishment of the skin barrier $[1,18]$.

Although the morphological and developmental changes during embryogenesis are wellcharacterised at the histological level, understanding at the molecular level is less comprehensive. The interactions of conflicting growth factors, signalling cascades and downstream transcriptional programs play a critical role in coordination of development, but the diversity of cell types and the often transient nature of such interactions renders these processes challenging to investigate. More focussed data can be obtained by careful isolation of specific embryonic tissues, however not all tissues are tractable to such an approach, and restricting analysis in this fashion loses wider developmental context. Recent technological advances have permitted elegant studies of embryonic gene expression down to single-cell level $[19,20]$, suggesting that detailed study of the interactions between individual cell lineages might now be possible, however such approaches also represent a substantial undertaking both in time and resources. Furthermore, these approaches are not without limitation: the whole transcriptome nature of the method typically favours breadth over depth [21, 22]: large-scale changes involving many gene components will be mapped well, while more specific, smaller-scale processes can be missed. In particular, constraints in read depth mean low abundance transcripts can be under-represented in such datasets [23], and thus more conventional methods remain vital research tools.

Measurement of gene expression via qPCR (using cDNA prepared from extracted RNA) is a comparatively inexpensive and flexible means of measuring expression of specific gene targets, including rare transcripts expressed at low levels or restricted to minority cell populations. Given the quantitative nature of this technique, normalisation of expression data is essential: RNA extraction efficiency, RNA integrity and cDNA synthesis efficiency all represent sources of variability that can potentially obscure genuine changes in gene expression, or create the appearance of change where none exists (best summarised in the 
MIQE guidelines, [24]). Normalisation can be conducted using reference genes: genes known to be stably expressed under the conditions studied. RNA is an inherently labile molecule, however, and cellular turnover at the transcript level is often both more dramatic, and more rapid, than at the protein level. Genes considered broadly appropriate for protein normalisation such as GAPDH and ACTB (beta-actin) are historically popular choices, but we and others have shown these often perform poorly as references [25-31]. 18S ribosomal RNA is also used widely, but this necessitates use of random priming in cDNA preparation (rRNA lacks polyA tails, thus oligo dT priming will not reverse transcribe 18S). Synthesis and degradation of ribosomal RNA also differs from that of mRNA [32], and the sheer abundance of rRNA sequences could mask marked changes in the mRNA pool (ribosomes represent $\sim 80-90 \%$ of total cellular RNA, while mRNA accounts for $\sim 5 \%$, thus mRNA levels could change by a factor of two or more without significantly altering measured rRNA content). Crucially, reference genes suitable for one comparative scenario are not guaranteed to be appropriate for another, and identification of reference genes appropriate for the conditions studied represents a key step in any qPCR-based investigation. Identification of appropriate reference genes a priori is challenging, and various mathematical approaches exist: the geNorm [33], Normfinder [34], Bestkeeper [35] and deltaCt [36] methods all require a representative collection of cDNA samples, and a broad panel of candidate reference genes, but each assesses suitability via subtly different criteria (see supplementary data for an overview). Combining these complementary approaches increases the power of investigations: individual rankings might differ between methods, but truly strong candidates should consistently score highly regardless of assessment method (and discrepancies between methods can moreover highlight interesting biological information, as reported previously [26, 37]).

The developing embryo represents an especially dynamic transcriptional environment, transcriptional changes are likely to be dramatic as development proceeds, making determination of reference genes especially critical; such developmental changes might be even more profound in a mammal with a short gestation like the mouse. Several studies have investigated appropriate references for earlier developmental stages, with a focus on early morula/blastocyst formation [38-40], or progression from blastocyst (E3.5) to mid organogenesis (E11.5) [41, 42], though these studies have produced conflicting results, with $A C T B$ scoring highly in some, but poorly in others (some investigators have reported that embryonic reference gene suitability might even vary by mouse strain [39]). Less attention has been dedicated to later stages of embryogenesis, where cell and organ lineages are more established, and developmental changes become consequently more focussed and tissue-specific. Several studies have been conducted on specific organs (such as the developing gonads [43], heart [44] or thymus [45]), but no specific study has addressed reference genes appropriate for normalisation at the whole embryo level at these later stages.

We have thus investigated reference genes appropriate for normalising gene expression in whole mouse embryos collected at E11.5, E12.5, E13.5, E14.5, E15.5, E16.5 and E18.5, spanning development from mid to late gestation. Given the marked developmental changes that occur within the head and the forelimbs over this period (Fig 1), we have also determined reference genes appropriate for these specific tissues (at E13.5, E16.5 and E18.5). Our collection of samples is substantial ( $\mathrm{N}=3-5$ per time point) and we have used a broad panel of 15 candidate reference genes (ACTB, 18S, SDHA, GAPDH, HTATSF1, 
CDC40, RPL13A, CSNK2A2, AP3D1, HPRT1, CYC1, EIF4A, UBC, B2M and PAK1IP1 Table 1). This panel includes candidates widely used historically (18S, GAPDH, ACTB), those that we have shown be strong references in mouse skeletal muscle (CSNK2A2, $A P 3 D 1, R P L 13 A)[25,37]$, and also shown by others to be viable references in rodent brains (UBC, HPRT1, RPL13A, 18S) [46, 47]. To maximise the power of our study, we have assessed reference gene suitability using all four algorithms described above (geNorm, deltaCt, BestKeeper and Normfinder).

\begin{tabular}{c|c}
$\begin{array}{c}\text { Gene } \\
\text { name }\end{array}$ & Full name/function \\
\hline ACTB & Beta-Actin: cytoskeletal component \\
18S & 18S ribosomal RNA: small ribosomal subunit \\
SDHA & Succinate Dehydrogenase subunit A: mitochondrial complex II \\
GAPDH & Glyceraldehyde 3-Phosphate Dehydrogenase: glycolysis pathway \\
HTATSF1 & HIV-1 Tat-specific factor 1: transcriptional elongation/splicing factor \\
CDC40 & Cell Division Cycle 40: cell cycle/spliceosome factor \\
RPL13a & Ribosomal Protein L13: component of large ribosomal subunit \\
CSNK2A2 & Casein Kinase 2 Alpha 2: subunit of casein kinase \\
AP3D1 & Adaptor Related Protein Complex 3 Subunit Delta 1: vesicle trafficking \\
HPRT1 & Hypoxanthine Phosphoribosyltransferase 1: purine salvage pathway \\
CYC1 & Cytochrome C1: mitochondrial complex III \\
EIF4A & Eukaryotic Initiation Factor 4A: helicase component of translation initiation \\
UBC & complex \\
B2M & Ubiquitin C \\
PAK1IP1 & Beta 2 Microglobulin: component of MHC class 1 complex
\end{tabular}

Table 1: list of candidate genes and their full names/functions

\section{Methods}

\section{Ethics statement}

A total of 70 mouse embryos (strain C57BL/10) were obtained post-mortem from 10 pregnant females: 35 embryos were used for this study (additional embryos were collected for a separate study). All mice were bred under UK Home Office Project Licence, approved by the Royal Veterinary College Animal Welfare and Ethical Review Body. Mice were held in individually ventilated cages in a minimal disease unit at an average $21^{\circ} \mathrm{C}$ in a 12 hours light/ 12 hours dark light cycle with food and water provided ad-lib. Mating trios (one male, 2 females) were set up and monitored each morning until all females had been mated (as shown by a vaginal plug). All pregnant females were killed by cervical dislocation at the required gestational age and all embryos were killed via hypothermia and exsanguination.

\section{Sample collection and study design}

Matings were assumed to occur at midnight, thus first detection of a vaginal plug was designated as 0.5 days post coitum, or E0.5, with subsequent days incremented accordingly 
(E1.5, E2.5 etc). Seven time points were used for whole embryo samples (E11.5, E12.5, E13.5, E14.5, E15.5, E16.5 and E18.5) with E13.5, E16.5 and E18.5 also used for head and forelimb samples. A minimum of three embryos were used for each time point, though where litter sizes permitted, greater numbers were collected (Whole embryos: E13.5 N=4, E18.5 $\mathrm{N}=5$; Heads: E18.5 N=5, for a total of 24 whole embryo samples, 11 head samples and 9 forelimb samples -see Supplementary Table S1). Given potential variation in precise mating, ovulation and conception times, gestational ages should be considered approximate, particularly where different litters are pooled for a single timepoint (E13.5, E14.5, E18.5). At the appropriate gestational stage (all sample collections were performed between midday and $2 \mathrm{pm}$ ), pregnant females were killed by cervical dislocation. Uterine horns were quickly removed and placed on ice (additional organs and muscles were collected from the adults for separate studies). After death, embryos were dissected from their uterine environments (including removal from amniotic sac) and either kept intact (whole embryos), or further dissected to isolate forelimbs (each sample used both forelimbs) and heads (remaining tissue from the body was also collected). All samples were snap-frozen in liquid nitrogen and stored at $-80^{\circ} \mathrm{C}$ until use.

\section{RNA isolation and qPCR}

Tissues (whole embryos, heads or forelimbs) were pulverised via dry-ice-cooled cell-crusher (Cellcrusher Ltd), and $\sim 100-200 \mathrm{mg}$ frozen tissue powder (well mixed to ensure representative sampling) was used to prepare RNA. RNA was isolated using TRIzol (Invitrogen) as described previously [26, 37], with inclusion of an additional chloroform extraction (1:1) after the phase separation step and inclusion of $10 \mu \mathrm{g}$ glycogen during precipitation to maximise RNA yield. RNA yield and purity were assessed via nanodrop (ND1000) and samples with 260/230 ratios below 1.7 were subjected to a second precipitation. All cDNA was prepared using the RTnanoscript2 kit (Primerdesign), using 1600 ng of RNA per reaction, with oligo dT and random nonamer priming. All reactions were subsequently diluted $1 / 20$ with nuclease-free water to minimise downstream PCR inhibition. qPCR reactions were performed in duplicate or triplicate in $10 \mu$ volumes using $2 \mu$ diluted cDNA ( 8ng cDNA per well assuming 1:1 conversion) in a CFX384 lightcycler using PrecisionPLUS SYBR green qPCR mastermix (Primerdesign), with a melt curve included as standard (Supplementary Figure $\mathrm{S} 1$ ). All $\mathrm{Cq}$ values were determined via linear regression. Primers to ACTB, SDHA, GAPDH, HTATSF1, CDC40, RPL13A, CSNK2A2, AP3D1, CYC1, EIF4A, UBC, B2M and PAK1IP1 were taken from the geNorm and geNorm PLUS kits (Primerdesign): all give efficiencies of $95-105 \%$ and produce single amplicons. Sequences are proprietary but anchor nucleotides and context sequences can be provided on request. Primers to $18 S$ were those used previously [25], and those to HPRT1 were the pan-species set (HPSF) validated by Valadan et al [48]. Primers targeting the 5 ' terminus of dystrophin dp71 were designed using Primer3 (https://primer3.ut.ee/). All sequences are provided below:

18S F 5'-GTAACCCGTTGAACCCCATT-3'

18S R 5'-CCATCCAATCGGTAGTAGCG-3'

HPSF F 5'-GGACTAATTATGGACAGGACTG-3' 
HPSF R 5'-GCTCTTCAGTCTGATAAAATCTAC-3'

Dp71 F 5'-GTGAAACCCTTACAACCATGAG-3'

Dp71 R 5'-CTTCTGGAGCCTTCTGAGC-3'

\section{Data analysis}

All data was analysed using Microsoft Excel, using four different reference gene analysis programs: geNorm [33], deltaCt [36], BestKeeper [35] and Normfinder [34] (see supplementary material). BestKeeper and deltaCt methods used mean $\mathrm{Cq}$ values, while for geNorm and Normfinder Cq values were first linearised to relative quantities (RQ). All data were analysed within a single dataset (44 samples) or as embryos (24 samples), heads (11) or forelimbs (9) alone. Normfinder analysis further allows designation of user-defined groups: accordingly, data was analysed ungrouped as for the other packages above, or grouped as follows. Whole dataset, grouped by tissue type or age; whole embryos, grouped by age; heads grouped by age; forelimbs grouped by age.

To integrate the outputs of all programs, the per-gene geometric mean was generated from the scores of each specific comparison (whole dataset, embryos, heads, forelimbs). Bestkeeper ranks genes by coefficient of correlation (where low values represent poor correlation), while all other methods rank by stability (were low values represent high stability). Accordingly, values for Bestkeeper were inverted (1-value), with any negative correlation coefficients first set to zero (to give a subsequent score of 1). GeNorm suggests a best pair: for comparative purposes, each of the best pair were assigned the same score. Data from Normfinder was ungrouped (grouped analysis was not used in the integrated assessment).

\section{Statistical analysis}

Statistical analysis was performed using Graphpad Prism 8.0 (Pearson correlations) or Microsoft Excel (coefficients of variation). Dataset coefficients of variation were determined for each timepoint individually, then summed to provide the overall variation.

\section{Results}

\section{Raw Cq values}

Raw Cq data (all genes, all samples) serves as a simple first-pass assessment of a reference gene panel. Most genes showed highly consistent expression across all samples (particularly $U B C$ ), however expression of $18 S$ was unexpectedly varied (Fig 2A): closer examination of the data suggested that levels of this gene varied not by age or tissue, but by cDNA synthesis batch, with one batch producing 18S Cqs 3 cycles later (corresponding to a roughly 10 -fold reduction in template). Preparation of fresh cDNA for these samples corrected this discrepancy (Fig 2B). Given the comparatively consistent expression for other genes in our panel, we investigated further: restriction to $18 \mathrm{~S}$ signal only implied a defect specifically in random priming. Random priming is not required for cDNA synthesis per se, but is necessary for reverse transcription of ribosomal RNAs (which lack polyA tails) and 
more importantly, to capture 5' sequence of longer mRNAs: deficient random priming would be expected to lower apparent expression of such mRNAs. To confirm this we measured expression of the dystrophin isoform dp71: this isoform is modestly expressed in the developing embryo, can only be distinguished from other dystrophin isoforms by primers targeting the unique first exon, and with a transcript $\sim 5 \mathrm{~kb}$ in length, capture of the 5 ' terminus requires random priming. As shown (Fig 2C), measured Cq values for this isoform showed a very similar pattern of batch-specific variation which was similarly corrected following preparation of fresh cDNA from these samples.

Assessment of our corrected dataset (Fig 2B) was largely in line with expected expression profiles, with known highly-abundant RNAs (18S, ACTB, GAPDH) showing the lowest mean Cq values. Interestingly, EIF4A, an RNA helicase that unwinds mRNA secondary structure for translational initiation, was the least abundant transcript in our panel. Given the fundamental role protein synthesis plays, and the high translational demands of a growing embryo, this finding was unexpected (though we note that even as the lowest expression gene in our panel, levels of this transcript were still comparatively high: mean Cq of 23.9). Studies have suggested that initiation via the elF4 protein complex involves a high degree of recycling at the 5' UTR, with the same complex being reused multiple times in rapid succession: initiation complexes might be in lower overall demand than genes associated with metabolism or kinase signalling.

\section{geNorm analysis}

The iterative geNorm algorithm ranks candidate genes by their mean pairwise variation $M$ (where high $\mathrm{M}$ represents high variation) to determine the 'best pair', the pair of genes that correlate most closely across all samples. Low $\mathrm{M}$ values thus represent more suitable genes, but there is no fixed threshold: conventionally, $\mathrm{M}$ values $<0.5$ are considered acceptable as reference genes, while for samples with innately variable expression patterns (such as different tissues) values $<1.0$ may be accepted. GeNorm analysis (Fig 3, Table 2) revealed high overall stability: assessment of our dataset as a whole (all samples, Fig 3A) revealed 14 of our 15 candidate genes had acceptable $M$ values $(\mathrm{M}<0.5) . C D C 40$ and HTATSF1 were the 'best pair', though RPL13A, PAK1IP1 and ACTB also performed well. $C Y C 1, G A P D H, B 2 M$ and HPRT1 all performed poorly, though only the latter failed to clear the $\mathrm{M}<0.5$ threshold. We repeated the analysis using specific subsets of our data (embryos, heads or forelimbs only: Fig $3 \mathrm{~B}, \mathrm{C}$ and $\mathrm{D}$ respectively). Embryo samples alone gave results very similar to the dataset as a whole, both in ranking and magnitude of stability: CDC40 and HTATSF1 were again the best pair, with RPL13A, PAK1IP1 and ACTB also scoring highly, while CYC1, GAPDH, B2M and HPRT1 scored poorly. In heads alone, CDC40 and HTATSF1 were also the best pair, with RPL13A and ACTB being similarly high scoring (though here GAPDH also performed well, while PAK1IP1 ranked poorly), but stability overall was greater in this tissue: all $M$ values were $<0.5$, with 8 of the 15 showing $M$ values $<0.2$. Greater overall stability was similarly revealed by analysis of forelimbs: a different best pair was obtained here ( $A C T B$ replacing CDC40), though CDC40 (along with RPL13a) remained high scoring.

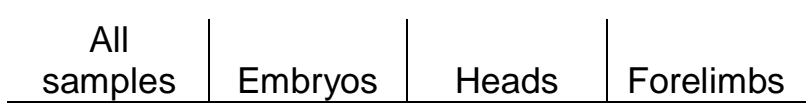




\begin{tabular}{cc|c|c|c} 
Best & HTATSF1 & HTATSF1 & HTATSF1 & ACTB \\
pair & $\mathbf{+}$ & $\mathbf{+}$ & $\mathbf{+}$ & $\mathbf{+}$ \\
& CDC40 & CDC40 & CDC40 & HTATSF1 \\
Most & ACTB & PAK1IP1 & ACTB & CDC40 \\
stable & RPL13a & RPL13a & RPL13a & RPL13a \\
& PAK1IP1 & ACTB & GAPDH & $18 S$ \\
& AP3D1 & AP3D1 & 18S & AP3D1 \\
& 18S & CSNK2A2 & UBC & EIF4A \\
& UBC & $18 S$ & PAK1IP1 & UBC \\
& CSNK2A2 & UBC & AP3D1 & PAK1IP1 \\
& EIF4A & EIF4A & CSNK2A2 & GAPDH \\
& SDHA & SDHA & CYC1 & CSNK2A2 \\
& CYC1 & CYC1 & SDHA & CYC1 \\
& GAPDH & GAPDH & EIF4A & SDHA \\
Least & B2M & B2M & B2M & HPRT1 \\
stable & HPRT1 & HPRT1 & HPRT1 & B2M
\end{tabular}

Table 2: geNorm rankings

GeNorm results for the entire dataset or tissue-specific subsets (as indicated), ranked from most stable to least stable. Genes with stability value $M>0.5$-poor scoring candidates- are indicated by italics.

Determination of a best pair does not imply that only two reference genes should be used: geNorm analysis also therefore determines the change in pairwise variation elicited by increasing the number of reference genes. Typically, variation below 0.2 is considered acceptable, and while our data showed addition of a third reference gene lowered variation, the best pair alone was sufficient in all cases (Supplementary Fig S2).

\section{DeltaCt analysis}

The DeltaCt $(\mathrm{dCt})$ method ranks genes by the mean standard deviation of their pairwise $\mathrm{Cq}$ differences, with lower values indicating more stable references. Using this method (Fig 4, Table 3), comparable results were obtained from analysis of the dataset as a whole, or restricted to embryos only: sorting by mean deltaCt standard deviation revealed HPRT1 and $B 2 M$ were the worst performing candidates in both cases, by a considerable margin. GAPDH, EIF4A and CYC1 also scored poorly, but modestly so: indeed, HPRT1 and B2M aside, increases in apparent stability across the panel were slight, suggesting most of our panel represent valid reference genes (as suggested by geNorm, above). AP3D1, PAK1IP1, CSNK2A2, RPL13A, UBC, CDC40 and HTATSF1 all scored highly: despite slight differences in ranking between the whole dataset and embryos alone, all six genes had very similar scores (ranging from 0.42-0.5). Restricting analysis to the head alone (Fig 4C) produced a similar pattern of ranking, and increased apparent stability across the entire panel. With the exception of HPRT1 and B2M, essentially every candidate gene represented a strong candidate for normalising gene expression in embryonic head tissues. HPRT1 and B2M also 
ranked poorest in forelimbs alone (Fig 4D), but here $18 S$ and EIF4A were much higher scoring, while $A C T B$ and $C D C 40$ were not, implying clear differences in expression patterns in these tissues (and a marked departure from geNorm, where ACTB formed part of the best pair).

\begin{tabular}{cc|c|c|c} 
& All & & & \\
Most & samples & Embryos & Heads & Forelimbs \\
\cline { 2 - 5 } stable & AP3D1 & AP3D1 & AP3D1 & 18S \\
& PAK1IP1 & CSNK2A2 & PAK1IP1 & AP3D1 \\
& UPC & RPL13a & CSNK2A2 & UBC \\
& RPL13a & PAK1IP1 & UBC & EIF4A \\
& CSNK2A2 & HTATSF1 & SDHA & PAK1IP1 \\
& CDC40 & CDC40 & RPL13a & CSNK2A2 \\
& HTATSF1 & UBC & CYC1 & HTATSF1 \\
& SDHA & SDHA & GAPDH & GAPDH \\
& 18S & ACTB & HTATSF1 & RPL13a \\
& ACTB & 18S & ACTB & CYC1 \\
& CYC1 & CYC1 & CDC40 & CDC40 \\
& EIF4A & EIF4A & 18S & SDHA \\
& GAPDH & GAPDH & EIF4A & ACTB \\
Least & B2M & B2M & B2M & HPRT1 \\
stable & HPRT1 & HPRT1 & HPRT1 & B2M
\end{tabular}

Table 3: DeltaCt rankings

DeltaCt results for the entire dataset or tissue-specific subsets (as indicated), ranked from most stable to least stable. Genes with mean dCt standard deviations $>0.6$-poor scoring candidates- are indicated by italics.

\section{Bestkeeper analysis}

The Bestkeeper method averages the entire presented dataset to generate a consensus profile: the 'Bestkeeper', and then ranks individual genes by correlation with this. As shown above, analysis via geNorm and $\mathrm{dCt}$ tended to be more effective at highlighting poor genes from our panel than at identifying consistently strong candidates. Bestkeeper analysis added to this emerging pattern (Fig 5, Table 4): most candidates performed well, with HPRT1 and $B 2 M$ remaining the exceptions (exhibiting negative correlation with the Bestkeeper in the case of heads and forelimbs). Rankings of higher scoring genes disagreed with previous analyses, however: the gene EIF4A -ranked lower by both geNorm and dCt- correlated well with a Bestkeeper derived from the whole dataset (or from embryos only), while ACTB, HTATSF1 and CDC40 correlated less closely. Interestingly, EIF4A also correlated closely with a Bestkeeper derived from forelimb data only (Fig 5D), but not one derived from head samples (Fig 5C), while CYC1 was high ranking in this latter tissue, but not in embryos, forelimbs or the dataset as a whole. $18 \mathrm{~S}$ was also closely correlated with the forelimb Bestkeeper (in agreement with $\mathrm{dCt}$ ) but performed poorly in the other dataset groupings. 


\begin{tabular}{cc|c|c|c} 
& $\begin{array}{c}\text { All } \\
\text { samples }\end{array}$ & $\begin{array}{c}\text { Whole } \\
\text { embryos }\end{array}$ & Heads & Forelimbs \\
\cline { 2 - 5 } Most & EIF4A & CSNK2A2 & AP3D1 & EIF4A \\
stable & AP3D1 & AP3D1 & SDHA & 18S \\
& SDHA & EIF4A & CYC1 & HTATSF1 \\
& UBC & SDHA & CSNK2A2 & ACTB \\
& GAPDH & GAPDH & PAK1IP1 & CDC40 \\
& PAK1IP1 & RPL13a & GAPDH & AP3D1 \\
& RPL13a & PAK1IP1 & HTATSF1 & UBC \\
& CSNK2A2 & UBC & RPL13a & RPL13a \\
& CDC40 & CDC40 & ACTB & GAPDH \\
& ACTB & HTATSF1 & CDC40 & PAK1IP1 \\
& HTATSF1 & ACTB & UBC & SDHA \\
& CYC1 & CYC1 & 18S & CSNK2A2 \\
& 18S & 18S & EIF4A & CYC1 \\
Least & HPRT1 & HPRT1 & HPRT1 & HPRT1 \\
stable & B2M & B2M & B2M & B2M
\end{tabular}

Table 4: Bestkeeper rankings

Bestkeeper results for the entire dataset or tissue-specific subsets (as indicated), ranked by Pearson correlation coefficient ( $r$ ) with the Bestkeeper, from most stable to least stable.

Genes with $r<0.6$-poor scoring candidates- are indicated by italics.

\section{Normfinder analysis}

Unlike the other three algorithms, Normfinder does not make pairwise comparisons, but instead assesses each gene for individual stability across the dataset. This method also allows two different analysis approaches: grouped and ungrouped. Ungrouped analysis considers the dataset without distinction between samples, while grouped analysis allows discrete subcategories to be defined within the dataset. Accordingly, we used ungrouped analysis to assess our combined dataset and tissue-restricted subsets (Fig 6, Table 5), with further grouped analysis to introduce age and tissue-type as subcategories within those datasets where appropriate. In some respects, ungrouped analysis agreed with the above methods (particularly dCt): B2M and HPRT1 were consistently ranked last (by a considerable margin), while many of the remaining genes had comparable high scores. EIF4A ranked lower here in all tissues but forelimbs, while CNSK2A2, PAK1IP1 and especially $A P 3 D 1$ all typically performed well (in agreement with $\mathrm{dCt}$ and geNorm, but in contrast to Bestkeeper). 18S was again ranked highest in forelimbs alone, but ACTB fared very poorly (in agreement with $\mathrm{dCt}$ and Bestkeeper, but not geNorm)

\begin{tabular}{cc|c|c|c} 
& $\begin{array}{c}\text { All } \\
\text { samples }\end{array}$ & $\begin{array}{c}\text { Whole } \\
\text { embryos }\end{array}$ & Heads & Forelimbs \\
\cline { 2 - 5 } Most & AP3D1 & AP3D1 & AP3D1 & 18S \\
stable & UBC & CSNK2A2 & CSNK2A2 & AP3D1
\end{tabular}




\begin{tabular}{cc|c|c|c} 
& CSNK2A2 & RPL13a & PAK1IP1 & UBC \\
& PAK1IP1 & UBC & SDHA & EIF4A \\
RPL13a & PAK1IP1 & CYC1 & PAK1IP1 \\
SDHA & SDHA & UBC & CSNK2A2 \\
& CDC40 & HTATSF1 & RPL13a & GAPDH \\
HTATSF1 & CDC40 & GAPDH & CYC1 \\
& CYC1 & CYC1 & HTATSF1 & RPL13a \\
& 18S & HCS & 18S & HTATSF1 \\
& ACTB & ACTB & ACTB & SDHA \\
& GAPDH & EIF4A & CDC40 & CDC40 \\
& EIF4A & GAPDH & EIF4A & HPRT1 \\
Least & B2M & B2M & B2M & ACTB \\
stable & HPRT1 & HPRT1 & HPRT1 & B2M
\end{tabular}

Table 5: Normfinder rankings (ungrouped)

Ungrouped Normfinder results for the entire dataset or tissue-specific subsets (as indicated), ranked from most stable to least stable. Genes with stability values $>0.5-$ poor scoring candidates- are indicated by italics.

Grouped analysis of our datasets (adding in an age or tissue component to the combined data, and an age component to tissue-specific subsets) differed from ungrouped in several respects (Fig 7, Table 6), suggesting interesting nuances of expression stability within our datasets. When the entire dataset was grouped by tissue (embryo, head, forelimb) no gene had a stability value greater than 0.2 , i.e. stability across the panel was substantially greater overall than for the ungrouped dataset, or the same data grouped by age. This suggests tissue-specific differences in gene expression might be mild (and certainly milder than agespecific changes). HPRT1, B2M, EIF4A and $18 S$ were the lowest ranked genes in both tissue and age-specific grouping, and indeed HPRT1 and B2M scored poorly in all rankings, with the marked exception of embryos grouped by age (Fig 7C). Here CYC1, PAK1IP1 and $18 S$ performed worse (exceptionally so, in the case of $18 S$ ). Grouping embryos by age moreover revealed much lower overall stability for the panel than all other grouped analyses: these findings imply global age-related changes are of greatest magnitude, and that CYC1, PAK1IP1 and 18S in particular might exhibit consistent age-associated changes. Grouped analysis additionally generates a best pair: two genes that may vary between groups, but in opposite directions (given greater overall stability than any individual gene, when combined). In most cases, this best pair did not feature the most stable genes, but as the majority of genes performed well under the majority of comparisons, this was not unexpected (and the stability advantages offered by this best pair were in most cases only modest improvements over the highest scoring candidates alone).

\begin{tabular}{cc|c|c|c|c} 
& \multicolumn{2}{c|}{ All samples } & $\begin{array}{c}\text { Whole } \\
\text { embryos } \\
\text { By age }\end{array}$ & $\begin{array}{c}\text { Heads } \\
\text { By age }\end{array}$ & $\begin{array}{c}\text { Forelimbs } \\
\text { By age }\end{array}$ \\
\cline { 2 - 6 } & By age & By tissue & By & BS \\
Best & $\mathbf{1 8 S}$ & CSNK2A2 & CSNK2A2 & CYC1 & $\mathbf{1 8 S}$ \\
pair & + & + & + & + & + \\
& SDHA & AP3D1 & AP3D1 & PAK1IP1 & UBC \\
\cline { 2 - 6 }
\end{tabular}




\begin{tabular}{cc|c|c|c|c} 
Most & AP3D1 & UBC & CSNK2A2 & AP3D1 & AP3D1 \\
stable & HTATSF1 & SDHA & EIF4A & CSNK2A2 & 18S \\
& CYC1 & PAK1IP1 & AP3D1 & CYC1 & UBC \\
& CSNK2A2 & CDC40 & RPL13a & PAK1IP1 & EIF4A \\
& RPL13a & CYC1 & HTATSF1 & SDHA & PAK1IP1 \\
& SDHA & AP3D1 & SDHA & UBC & GAPDH \\
& PAK1IP1 & RPL13a & ACTB & RPL13a & HTATSF1 \\
& UBC & ACTB & CDC40 & ACTB & CSNK2A2 \\
& CDC40 & GAPDH & HPRT1 & GAPDH & CYC1 \\
& ACTB & CSNK2A2 & GAPDH & HTATSF1 & SDHA \\
& GAPDH & HTATSF1 & UBC & 18S & ACTB \\
& 18S & $18 S$ & B2M & EIF4A & CDC40 \\
& EIF4A & HPRT1 & CYC1 & CDC40 & RPL13a \\
Least & B2M & EIF4A & PAK1IP1 & B2M & HPRT1 \\
stable & HPRT1 & B2M & 18S & HPRT1 & B2M
\end{tabular}

Table 6: Normfinder rankings (grouped)

Grouped Normfinder results for the entire dataset or tissue-specific subsets, grouped internally by age or tissue (as indicated), ranked from most stable to least stable. The best pair of genes are indicated separately (bold). Genes with stability values $>0.5-$ poor scoring candidates- are indicated by italics.

\section{Summary and Validation}

Despite the differences in highest scoring candidates between algorithms, all four analysis methods tended to suggest that many of our candidate genes represented stable references. In most cases, the highest-ranking candidate was only fractionally more stable than candidates ranked seventh or eighth. Conversely, at the other end of the stability spectrum all algorithms were in much greater agreement. Regardless of method used or tissue assessed, HPRT1 and B2M were near-universally ranked last, and often by a substantial margin, implying strongly that these genes are inappropriate choices. To illustrate this more clearly, we integrated our collected data in a manner similar to the RefFinder method developed by Xie et al [49], taking the geometric mean of all algorithm scores, either for the entire dataset, or for embryos, heads and forelimbs alone (see methods). When combined in this fashion (Fig 8, Table 7), the patterns and rankings are remarkably similar for all sets except forelimbs alone. In embryos, heads, or all samples together, AP3D1 is the strongest candidate, while RPL13A, PAK1IP1 and CSNK2A2 are also highly ranked. In forelimbs, EIF4A, 18S, ACTB and HTATSF1 are the strongest candidates, however AP3D1, RPL13A and $P A K 1 I P 1$ also score highly in this tissue (while CSNK2A2 does not). Taken together, these data suggest that $A P 3 D 1, P A K 1 I P 1$ and $R P L 13 A$ might represent a universally suitable panel.

\begin{tabular}{cc|c|c|c} 
& $\begin{array}{c}\text { All } \\
\text { samples }\end{array}$ & $\begin{array}{c}\text { Whole } \\
\text { embryos }\end{array}$ & Heads & Forelimbs \\
\cline { 2 - 5 } Most & AP3D1 & AP3D1 & AP3D1 & EIF4A
\end{tabular}




\begin{tabular}{lc|c|c|c} 
stable & UBC & CSNK2A2 & CSNK2A2 & 18S \\
& PAK1IP1 & RPL13a & PAK1IP1 & HTATSF1 \\
& RPL13a & PAK1IP1 & SDHA & ACTB \\
& SDHA & CDC40 & CYC1 & AP3D1 \\
& CSNK2A2 & SDHA & HTATSF1 & CDC40 \\
& CDC40 & EIF4A & RPL13a & UBC \\
& EIF4A & HTATSF1 & GAPDH & RPL13a \\
& HTATSF1 & UBC & UBC & PAK1IP1 \\
& ACTB & ACTB & ACTB & GAPDH \\
& GAPDH & GAPDH & CDC40 & CSNK2A2 \\
& CYC1 & CSS & 18S & SDHA \\
& LSS & CYC1 & EIF4A & CYC1 \\
Least & HPRT1 & B2M & B2M & HPRT1 \\
stable & B2M & HPRT1 & HPRT1 & B2M
\end{tabular}

Table 7: Integrated ranking

Reference genes ranked by geometric mean of geNorm, dCt, Bestkeeper and Normfinder scores. Genes with stability values $>0.5$-poor scoring candidates- are indicated by italics.

For validation, we employed an approach we have used previously [26, 37]: using our high scoring candidates (AP3D1, RPL13A, PAK1IP1) to normalise our lowest scoring candidates (HPRT1 and B2M). Raw data for these genes suggested modest expression with a dramatic increase at E18.5, however following normalisation both genes show a progressive increase in expression with increasing age, whether in whole embryos, heads, or limbs (Fig 9). Normalisation would also be expected to reduce the overall coefficient of variation (CoV), and as shown this was indeed the case.

This validation method can be extended: the approaches used by each algorithm are subtly different, and thus information can be gleaned by examining cases where candidate rankings disagree. GeNorm, a method that ranks genes by pairwise variation, suggested HTATSF1 and CDC40 as the best pair near-unanimously, while these two genes performed only modestly under most other assessments. The implication is that these genes share a very similar pattern of expression, but in a manner unstable across samples. To investigate this directly, we used our high scoring candidates to normalise expression of HTATSF1 and CDC40. Raw data was indeed highly variable between samples, and between tissues and ages, yet this variability was well-matched between the two genes, confirming their geNorm score (Fig 10). Following normalisation (which again substantially lowered CoV), this variability resolved into a mild, but remarkably consistent, decrease in expression with increasing age, in all tissues. Normalisation of CYC1, UBC or EIF4A (Supplementary Fig S3) gave similar results: $C Y C 1$, which typically scored highly in heads but low elsewhere, was revealed to be very consistently expressed in the former tissue while exhibiting ageassociated increases in embryos and forelimbs (Supplementary Fig S3A and B); UBC, which was ranked modestly overall, exhibited a similar age-associated increase in expression in all tissues except the head (Supplementary Fig S3C and D); EIF4A, which scored particularly highly in forelimbs, was indeed stable in this tissue, while showing age-specific increases in 
heads and highly variable behaviour in whole embryos (Supplementary Fig S3E and F).

Given $18 S$ and EIF4A were the highest scoring candidates in forelimbs, we further compared normalisation using these two genes with that obtained using the three genes above.

Normalised forelimb expression of B2M, HPRT1, CDC40 and HTATSF1 was comparable with either reference gene combination, though overall CoV values were lower using $A P 3 D 1$, RPL13A and PAK1IP1 (Supplementary Fig S4).

Finally, use of all three reference genes might not be necessary: while the MIQE guidelines suggest use of two reference genes at a minimum [24], our geNorm analysis suggested no substantial benefit in increasing number of genes from 2 to 3 (or indeed from 3 to 4).

Accordingly, we compared a normalisation factor (NF) using all three genes to one using $A P 3 D 1$ with either RPL13A or PAK1IP1 alone: the three gene NF was essentially identical to either 2-gene NF (gradients of 1.00 and 0.978 respectively, both with Pearson correlations of 0.99 -Supplementary Fig S5), suggesting that two genes (AP3D1 plus one other) are indeed sufficient.

\section{Discussion}

The later stages of embryonic development involve many significant changes: substantial organogenesis occurs over this period, as does formation of skeletal muscle and the skeleton itself. Within the head, the brain matures from a comparatively simple tube to an intricately subdivided organ complete with discrete ventricles and regional specialisation, the teeth develop from buds to near-maturity, while the eye progresses from the simple optic cup stage to a defined globe complete with lens and iris, hidden beneath the fused eyelids. Normalisation of gene expression throughout this period might well be expected to be challenging, however our data unexpectedly suggests otherwise. For our dataset, whether assessed in its entirety, or restricted to whole embryos, heads or forelimbs alone, all four analysis methods suggested that a substantial number of our candidate genes would serve as suitable reference genes: with the exception of HPRT1 and B2M, essentially any gene from our panel represents an adequate reference. Given our panel consists of genes specifically selected for their reported stability, this is perhaps more reassuring than not, however we note that use of a near-identical panel of genes in skeletal muscle revealed markedly lower overall stability (for geNorm in particular, only ACTB and RPL13a gave M values $<0.5$ ) [37]. Our findings here imply that the developing embryo might be more transcriptionally consistent than different mature skeletal muscles. Even with most genes scoring highly there were differences, however, both between algorithms and between tissues, but the combination of AP3D1, RPL13A and PAK1IP1 (or indeed AP3D1 plus one of the others) emerged as consistently stable overall, and thus represent universal reference genes for the developing mouse embryo. We note that $18 S$ and EIF4A apparently represent better candidates in forelimbs specifically, but only fractionally so: studies addressing developmental changes in limbs alone might technically be better served by these two, but this would necessarily sacrifice the utility advantages offered by a universal panel (and AP3D1, RPL13A and PAK1IP1 perform comparably to 18S/EIF4A in forelimbs Supplementary Fig S4).

AP3D1 codes for a component of the adaptor complex, which mediates non-clathrin-coated vesicle trafficking: we have previously found this gene to be stable throughout myogenic differentiation in culture [25] and in mature mouse skeletal muscle (healthy and dystrophic) 
[37], and our data here support the possibility that this gene might exhibit high overall stability in mouse. RPL13A codes for a protein component of the small ribosomal subunit: ribosomal proteins are ubiquitous, and some have proposed that such ubiquity renders them generally suitable as references [50], though this remains contentious [51]. As with AP3D1, we have previously found this gene to be stable in mature mouse skeletal muscle, and indeed also suitable as a reference in canine skeletal muscle (again, both healthy and dystrophic [26]). PAK1IP1 encodes a negative regulator of PAK1 kinase, and its presence in our top scoring candidates represents something of a surprise: this gene was included in our candidate panels for both myogenic cell cultures and mature mouse muscle [25, 37], and in those scenarios its performance was near-uniformly mediocre. Here this gene was ranked lower even than HPRT1 and B2M in Normfinder analysis of embryos grouped by age, but outside of this specific scenario the gene performed well, and as shown (Supplementary Fig S5), RPL13A and PAK1IP1 are essentially interchangeable when used in combination with AP3D1.

Our validation process confirmed the utility of these genes, and also revealed insight into changes in gene expression during development: normalised expression of HPRT1 and B2M (our lowest scoring genes) exhibited clear age-associated upregulation (Fig 9). B2M (beta 2 microglobulin) is a component of the MHC class I complex, and while this complex is present even at the earliest stages of development, studies in the rat have shown marked increases in expression in specific tissues such as skin, lung and inter-organ connective tissue [52]. Many of these tissues are only emerging at the earliest stages of our sample set (E11.5E13.5) and indeed the skin barrier is complete only toward the end of development (E16.5$E 18.5)$ [18]. A developmentally associated upregulation in B2M is consistent with these changes. HPRT1 encodes Hypoxanthine Phosphoribosyltransferase 1, a central component of the purine salvage pathway: this gene is stably expressed under many circumstances (we have reported this gene to be stable in healthy and dystrophic canine muscle [26]).

Mutations in this gene however cause the neurodevelopmental condition, Lesch-Nyhan disease, and the gene itself plays a key role in glial/neuronal fate choice [53], implying that expression is developmentally regulated. Our data supports this, and indeed suggests that expression of this gene increases earlier in the head than within the embryo as a whole (Fig 9B, expression at E16.5). Conversely, both CDC40 and HTATSF1 displayed age-correlated downregulation following normalisation (Fig 10): this decrease was modest (insufficient to exclude them as acceptable, though not optimal, references) and moreover was sufficiently consistent between the two genes as to flag them as the best pair under geNorm analysis. This finding serves as a prominent reminder of the potential risks in relying on a single reference gene analysis method, but also indicates these genes might be co-ordinately regulated. HTATSF1 encodes HIV-TAT specific factor 1, an RNA binding protein. Recent studies in embryonic stem cells implicate this factor in both regulation of ribosomal RNA processing and splicing of mRNA for ribosomal proteins, influencing protein synthesis and cell differentiation as a consequence [54]. CDC40 (cell division cycle 40, also known as PRP17, or pre-mRNA processing factor 17) encodes a protein component of the spliceosome, and is also involved in progression through the cell cycle. Given our data, it seems likely that a shared involvement in mRNA splicing underpins the remarkably wellmatched expression of these two genes, with requirements for spliceosomal components decreasing gradually with developmental progress. 
Our dataset also revealed a marked age-related increase in CYC1 expression in all tissues except the head (Supplementary Fig S3B). This gene encodes the mitochondrial electron transfer chain component cytochrome $\mathrm{C}$, and our findings are thus best explained by developmental mitochondrial biogenesis. The most likely source of such marked increases in mitochondrial content is skeletal muscle: E11.5 represents the approximate midpoint of primary myogenesis, in which small numbers of myofibres are laid down to serve as scaffolds for subsequent musculature, while the period from E14.5-E18.5 spans secondary myogenesis, a phase of more substantial muscle synthesis [10]. This latter phase might well be associated with a dramatic increase in mitochondrial content, both in the embryo as a whole but especially in the forelimb where skeletal muscle comprises a greater fraction of the total tissue. The head is comparatively muscle-poor by contrast, and craniofacial muscles are moreover derived from a different progenitor pool which need not mature along identical timescales [55]. The brain is a highly energetic tissue, however neurodevelopmental changes over this period are predominantly structural/differentiation rather than bulk increases in tissue mass. In contrast, EIF4A showed the reverse trend (Supplementary Fig S3F), increasing in heads but not forelimbs or embryos as a whole. This gene codes for an RNA helicase that unwinds 5' secondary structure for the translational preinitiation complex, and increases in expression in the head over this period might reflect the demands of the diverse transcription/translation programs necessary for brain maturation.

Finally, our experience with $18 S$ merits additional mention. Ribosomal RNAs comprise the vast bulk of total RNA ( $80-85 \%)$, but the absence of polyA tails necessitates random priming for conversion to cDNA. Oligo dT does not contribute to $18 \mathrm{~S}$ cDNA, thus this ribosomal target effectively serves as an internal control for efficiency of random priming. As we show here, batch-dependent variance in $18 S$ specifically (i.e. other genes remain comparable) is highly indicative of a failure in this specific step, perhaps due to degradation of random primer stocks. Under many circumstances, this might be of little consequence: while the processivity of reverse transcriptase is such that even highly optimised enzymes seldom incorporate more than 1500 nucleotides in a single binding event [56], the majority of mRNAs are of modest ( 3kb) length [57] and can typically be reverse-transcribed in their entirety from initial oligo dT priming alone. 5' sequence of longer transcripts $(5 \mathrm{~kb}+)$ will however be increasingly underrepresented or even absent under such conditions, and random priming is thus essential for qPCR studies investigating such transcripts (such as the early embryonic dystrophin isoform dp71). Critically, a failure in random priming will not be immediately apparent if all reference genes used can be successfully reverse-transcribed via oligo dT: our data suggests that for studies using random priming in combination with oligo $\mathrm{dT}$, particularly those investigating expression of long genes, measurement of $18 \mathrm{~S}$ could represent a prudent quality check regardless of its efficacy as a reference.

\section{Conclusion}

We have investigated potential reference genes for normalising quantitative PCR expression data in the developing mouse embryo from E11.5 to E18.5. We investigated expression both in whole embryos, or heads or forelimbs in isolation. Our data suggests that normalisation of expression over this period is not only possible, but that many reference genes are acceptable, though the genes AP3D1, RPL13A and PAK1IP1 are the strongest candidates overall. Our data suggests only a pair of genes is necessary for effective normalisation: 
$A P 3 D 1$ and one other. We and others have reported $R P L 13 A$ to be a strong candidate under other scenarios [26, 37, 58-60], while literature for PAK1IP1 is more limited: AP3D1 plus $R P L 13 A$ thus seems the most practical pair for normalising gene expression in the developing mouse embryo.

\section{Acknowledgements}

This work was funded by the Wellcome Trust (Grant number 101550, awarded to RJP) and the Gill Malone Memorial Award (to JCWH). This manuscript has been approved by the RVC research office and assigned the following number: CSS_02293

\section{Author contributions}

JCWH: Conceptualization, Formal Analysis, Funding acquisition, Investigation, visualization, Writing -original draft; DJW: Investigation, Resources, Supervision, Writing -review \& editing; RJP: Funding acquisition, Supervision, Writing -review \& editing.

\section{References}

1. Kaufman MH. The Atlas of Mouse Development. 1st ed: Academic Press; 1992.

2. Hill M. Embryology. Available from:

https://embryology.med.unsw.edu.au/embryology/index.php/Main Page.

3. Macdonald DW. The Encyclopedia of Mammals. 3rd ed: Oxford University Press; 2007.

4. Rossant J, Tam PPL. Blastocyst lineage formation, early embryonic asymmetries and axis

patterning in the mouse. Development (Cambridge, England). 2009;136(5):701-13. doi:

10.1242/dev.017178.

5. Tam PPL, Behringer RR. Mouse gastrulation: the formation of a mammalian body plan.

Mechanisms of Development. 1997;68(1-2):3-25. doi: 10.1016/s0925-4773(97)00123-8.

6. Maroto M, Bone RA, Dale JK. Somitogenesis. Development (Cambridge, England).

2012;139(14):2453-6. Epub 2012/06/28. doi: 10.1242/dev.069310. PubMed PMID: 22736241;

PubMed Central PMCID: PMCPMC3928720.

7. Taher L, Collette NM, Murugesh D, Maxwell E, Ovcharenko I, Loots GG. Global gene expression analysis of murine limb development. PLoS One. 2011;6(12):e28358. Epub 2011/12/17. doi: 10.1371/journal.pone.0028358. PubMed PMID: 22174793; PubMed Central PMCID: PMCPMC3235105.

8. Pollard AS, McGonnell IM, Pitsillides AA. Mechanoadaptation of developing limbs: shaking a leg. J Anat. 2014;224(6):615-23. Epub 2014/03/19. doi: 10.1111/joa.12171. PubMed PMID: 24635640; PubMed Central PMCID: PMCPMC4025889.

9. Pitsillides AA. Early effects of embryonic movement: 'a shot out of the dark'. J Anat. 2006;208(4):417-31. doi: 10.1111/j.1469-7580.2006.00556.x. PubMed PMID: 16637868.

10. Chal J, Pourquié O. Making muscle: skeletal myogenesisin vivoandin vitro. Development (Cambridge, England). 2017;144(12):2104-22. doi: 10.1242/dev.151035.

11. Buckingham M, Bajard L, Chang T, Daubas P, Hadchouel J, Meilhac S, et al. The formation of skeletal muscle: from somite to limb. J Anat. 2003;202(1):59-68. Epub 2003/02/18. doi: 
10.1046/j.1469-7580.2003.00139.x. PubMed PMID: 12587921; PubMed Central PMCID: PMCPMC1571050.

12. Chen VS, Morrison JP, Southwell MF, Foley JF, Bolon B, Elmore SA. Histology Atlas of the Developing Prenatal and Postnatal Mouse Central Nervous System, with Emphasis on Prenatal Days E7.5 to E18.5. Toxicol Pathol. 2017;45(6):705-44. Epub 2017/09/12. doi: 10.1177/0192623317728134. PubMed PMID: 28891434; PubMed Central PMCID: PMCPMC5754028. 13. Gaete M, Lobos N, Torres-Quintana MA. Mouse tooth development time sequence determination for the ICR/Jcl strain. J Oral Sci. 2004;46(3):135-41. Epub 2004/10/29. doi: 10.2334/josnusd.46.135. PubMed PMID: 15508745.

14. Heavner W, Pevny L. Eye development and retinogenesis. Cold Spring Harb Perspect Biol. 2012;4(12). Epub 2012/10/17. doi: 10.1101/cshperspect.a008391. PubMed PMID: 23071378; PubMed Central PMCID: PMCPMC3504437.

15. Sugiyama D, Inoue-Yokoo T, Fraser ST, Kulkeaw K, Mizuochi C, Horio Y. Embryonic regulation of the mouse hematopoietic niche. ScientificWorldJournal. 2011;11:1770-80. Epub 2011/11/30. doi: 10.1100/2011/598097. PubMed PMID: 22125435; PubMed Central PMCID: PMCPMC3201691.

16. de Boer BA, van den Berg G, de Boer PA, Moorman AF, Ruijter JM. Growth of the developing mouse heart: an interactive qualitative and quantitative 3D atlas. Developmental biology.

2012;368(2):203-13. Epub 2012/05/24. doi: 10.1016/j.ydbio.2012.05.001. PubMed PMID: 22617458. 17. Scott RP, Maezawa Y, Kreidberg J, Quaggin SE. Embryology of the Kidney: Elsevier; 2016. 18. Schmitz A, Lazi'c E, Koumaki D, Kuonen F, Verykiou S, Rubsam M. Assessing the in vivo epidermal barrier in mice: dye penetration assays. J Invest Dermatol. 2015;135(2):1-4. Epub 2015/01/13. doi: 10.1038/jid.2014.495. PubMed PMID: 25573050.

19. Cao J, Spielmann M, Qiu X, Huang X, Ibrahim DM, Hill AJ, et al. The single-cell transcriptional landscape of mammalian organogenesis. Nature. 2019;566(7745):496-502. Epub 2019/02/23. doi: 10.1038/s41586-019-0969-x. PubMed PMID: 30787437; PubMed Central PMCID: PMCPMC6434952. 20. He P, Williams BA, Trout D, Marinov GK, Amrhein H, Berghella L, et al. The changing mouse embryo transcriptome at whole tissue and single-cell resolution. Nature. 2020;583(7818):760-7. Epub 2020/07/31. doi: 10.1038/s41586-020-2536-x. PubMed PMID: 32728245; PubMed Central PMCID: PMCPMC7410830.

21. Zhang MJ, Ntranos V, Tse D. Determining sequencing depth in a single-cell RNA-seq experiment. Nat Commun. 2020;11(1):774. Epub 2020/02/09. doi: 10.1038/s41467-020-14482-y. PubMed PMID: 32034137; PubMed Central PMCID: PMCPMC7005864.

22. Baccarella A, Williams CR, Parrish JZ, Kim CC. Empirical assessment of the impact of sample number and read depth on RNA-Seq analysis workflow performance. BMC Bioinformatics. 2018;19(1):423. Epub 2018/11/16. doi: 10.1186/s12859-018-2445-2. PubMed PMID: 30428853; PubMed Central PMCID: PMCPMC6234607.

23. Liu Y, Ferguson JF, Xue C, Silverman IM, Gregory B, Reilly MP, et al. Evaluating the impact of sequencing depth on transcriptome profiling in human adipose. PLoS One. 2013;8(6):e66883. Epub 2013/07/05. doi: 10.1371/journal.pone.0066883. PubMed PMID: 23826166; PubMed Central PMCID: PMCPMC3691247.

24. Bustin SA, Benes V, Garson JA, Hellemans J, Huggett J, Kubista M, et al. The MIQE guidelines: minimum information for publication of quantitative real-time PCR experiments. Clinical chemistry. 2009;55(4):611-22. Epub 2009/02/28. doi: 10.1373/clinchem.2008.112797. PubMed PMID: 19246619.

25. Hildyard JC, Wells DJ. Identification and validation of quantitative PCR reference genes suitable for normalizing expression in normal and dystrophic cell culture models of myogenesis. PLoS currents. 2014;6. Epub 2014/03/19. doi:

10.1371/currents.md. faafdde4bea8df4aa7d06cd5553119a6. PubMed PMID: 24634799; PubMed Central PMCID: PMCPmc3948689.

26. Hildyard JCW, Taylor-Brown F, Massey C, Wells DJ, Piercy RJ. Determination of qPCR Reference Genes Suitable for Normalizing Gene Expression in a Canine Model of Duchenne Muscular 
Dystrophy. J Neuromuscul Dis. 2018;5(2):177-91. Epub 2018/04/05. doi: 10.3233/JND-170267. PubMed PMID: 29614692.

27. Panina Y, Germond A, Masui S, Watanabe TM. Validation of Common Housekeeping Genes as Reference for qPCR Gene Expression Analysis During iPS Reprogramming Process. Sci Rep. 2018;8(1):8716. Epub 2018/06/09. doi: 10.1038/s41598-018-26707-8. PubMed PMID: 29880849; PubMed Central PMCID: PMCPMC5992140.

28. Glare EM, Divjak M, Bailey MJ, Walters EH. beta-Actin and GAPDH housekeeping gene expression in asthmatic airways is variable and not suitable for normalising mRNA levels. Thorax. 2002;57(9):765-70. doi: 10.1136/thorax.57.9.765.

29. Stoecklin G, Sikand K, Singh J, Ebron JS, Shukla GC. Housekeeping Gene Selection Advisory: Glyceraldehyde-3-Phosphate Dehydrogenase (GAPDH) and $\beta$-Actin Are Targets of miR-644a. PLoS ONE. 2012;7(10). doi: 10.1371/journal.pone.0047510.

30. Lin J, Redies C. Histological evidence: housekeeping genes beta-actin and GAPDH are of limited value for normalization of gene expression. Development Genes and Evolution.

2012;222(6):369-76. doi: 10.1007/s00427-012-0420-x.

31. Nazari F, Parham A, Maleki AF. GAPDH, $\beta$-actin and $\beta 2$-microglobulin, as three common reference genes, are not reliable for gene expression studies in equine adipose- and marrow-derived mesenchymal stem cells. Journal of Animal Science and Technology. 2015;57(1). doi:

10.1186/s40781-015-0050-8.

32. Lafontaine DLJ. A garbage can for ribosomes: how eukaryotes degrade their ribosomes. Trends in biochemical sciences. 2010;35(5):267-77.

33. Vandesompele J, De Preter K, Pattyn F, Poppe B, Van Roy N, De Paepe A, et al. Accurate normalization of real-time quantitative RT-PCR data by geometric averaging of multiple internal control genes. Genome Biology. 2002;3(7):research0034.1 - research.11. PubMed PMID: doi:10.1186/gb-2002-3-7-research0034.

34. Andersen CL, Jensen JL, Ørntoft TF. Normalization of Real-Time Quantitative Reverse Transcription-PCR Data: A Model-Based Variance Estimation Approach to Identify Genes Suited for Normalization, Applied to Bladder and Colon Cancer Data Sets. Cancer Research. 2004;64(15):524550. doi: 10.1158/0008-5472.can-04-0496.

35. Pfaffl M, Tichopad A, Prgomet C, Neuvians T. Determination of stable housekeeping genes, differentially regulated target genes and sample integrity: BestKeeper - Excel-based tool using pairwise correlations. Biotechnology Letters. 2004;26(6):509-15. doi: 10.1023/b:bile.0000019559.84305.47.

36. Silver N, Best S, Jiang J, Thein SL. Selection of housekeeping genes for gene expression studies in human reticulocytes using real-time PCR. BMC molecular biology. 2006;7:33. Epub 2006/10/10. doi: 10.1186/1471-2199-7-33. PubMed PMID: 17026756; PubMed Central PMCID: PMCPMC1609175.

37. Hildyard JCW, Finch AM, Wells DJ. Identification of qPCR reference genes suitable for normalizing gene expression in the $\mathrm{mdx}$ mouse model of Duchenne muscular dystrophy. PLoS One. 2019;14(1):e0211384. Epub 2019/01/31. doi: 10.1371/journal.pone.0211384. PubMed PMID: 30699165; PubMed Central PMCID: PMCPMC6353192.

38. Gu Y, Shen X, Zhou D, Wang Z, Zhang N, Shan Z, et al. Selection and expression profiles of reference genes in mouse preimplantation embryos of different ploidies at various developmental stages. PLoS One. 2014;9(6):e98956. Epub 2014/06/14. doi: 10.1371/journal.pone.0098956.

PubMed PMID: 24927500; PubMed Central PMCID: PMCPMC4057156.

39. Jeong JK, Kang MH, Gurunathan S, Cho SG, Park C, Seo HG, et al. Evaluation of reference genes in mouse preimplantation embryos for gene expression studies using real-time quantitative RT-PCR (RT-qPCR). BMC Res Notes. 2014;7:675. Epub 2014/09/27. doi: 10.1186/1756-0500-7-675. PubMed PMID: 25256308; PubMed Central PMCID: PMCPMC4181407.

40. Mamo S, Gal AB, Bodo S, Dinnyes A. Quantitative evaluation and selection of reference genes in mouse oocytes and embryos cultured in vivo and in vitro. BMC developmental biology. 
2007;7:14. Epub 2007/03/08. doi: 10.1186/1471-213X-7-14. PubMed PMID: 17341302; PubMed Central PMCID: PMCPMC1832186.

41. Willems E, Mateizel I, Kemp C, Cauffman G, Sermon K, Leyns L. Selection of reference genes in mouse embryos and in differentiating human and mouse ES cells. Int J Dev Biol. 2006;50(7):62735. Epub 2006/08/08. doi: 10.1387/ijdb.052130ew. PubMed PMID: 16892176.

42. Barratt KS, Diamand KEM, Arkell RM. Identification of reference genes suitable for RT-qPCR studies of murine gastrulation and patterning. Mammalian Genome. 2018;29(9-10):656-62. doi: 10.1007/s00335-018-9769-0.

43. van den Bergen JA, Miles DC, Sinclair AH, Western PS. Normalizing Gene Expression Levels in Mouse Fetal Germ Cells1. Biology of Reproduction. 2009;81(2):362-70. doi: 10.1095/biolreprod.109.076224.

44. Ruiz-Villalba A, Mattiotti A, Gunst QD, Cano-Ballesteros S, van den Hoff MJB, Ruijter JM. Reference genes for gene expression studies in the mouse heart. Scientific Reports. 2017;7(1). doi: 10.1038/s41598-017-00043-9.

45. Halouani A, Jmii H, Michaux H, Renard C, Martens H, Pirottin D, et al. Housekeeping Gene Expression in the Fetal and Neonatal Murine Thymus Following Coxsackievirus B4 Infection. Genes. 2020;11(3). doi: 10.3390/genes11030279.

46. Ramhoj L, Axelstad M, Svingen T. Validation of endogenous reference genes in rat cerebral cortex for RT-qPCR analyses in developmental toxicity studies. PeerJ. 2019;7:e7181. Epub 2019/07/11. doi: 10.7717/peerj.7181. PubMed PMID: 31289700; PubMed Central PMCID: PMCPMC6599449.

47. Kang Y, Wu Z, Cai, Lu B. Evaluation of reference genes for gene expression studies in mouse and N2a cell ischemic stroke models using quantitative real-time PCR. BMC Neurosci. 2018;19(1):3. Epub 2018/02/03. doi: 10.1186/s12868-018-0403-6. PubMed PMID: 29390963; PubMed Central PMCID: PMCPMC5795833.

48. Valadan R, Hedayatizadeh-Omran A, Alhosseini-Abyazani MN, Amjadi O, Rafiei A, Tehrani M, et al. Data supporting the design and evaluation of a universal primer pair for pseudogene-free amplification of HPRT1 in real-time PCR. Data in Brief. 2015;4:384-9. doi: 10.1016/j.dib.2015.06.009. PubMed PMID: PMC4510554.

49. Xie F, Xiao P, Chen D, Xu L, Zhang B. miRDeepFinder: a miRNA analysis tool for deep sequencing of plant small RNAs. Plant Mol Biol. 2012;(1573-5028 (Electronic)). Epub 2012/02/01. doi: 10.1007/s11103-012-9885-2. PubMed PMID: 22290409.

50. Hsiao LL, Dangond F, Yoshida T, Hong R, Jensen RV, Misra J, et al. A compendium of gene expression in normal human tissues. Physiol Genomics. 2001;7(2):97-104. Epub 2002/01/05. doi: 10.1152/physiolgenomics.00040.2001. PubMed PMID: 11773596.

51. Thorrez L, Van Deun K, Tranchevent LC, Van Lommel L, Engelen K, Marchal K, et al. Using ribosomal protein genes as reference: a tale of caution. PLoS One. 2008;3(3):e1854. Epub 2008/03/28. doi: 10.1371/journal.pone.0001854. PubMed PMID: 18365009; PubMed Central PMCID: PMCPMC2267211.

52. Grabowska A, Lampson LA. MHC expression in nonlymphoid tissues of the developing embryo: Strongest class I or class II expression in separate populations of potential antigenpresenting cells in the skin, lung, gut, and inter-organ connective tissue. Developmental \& Comparative Immunology. 1995;19(5):425-50. doi: 10.1016/0145-305x(95)00003-c.

53. Kang TH, Park Y, Bader JS, Friedmann T. The housekeeping gene hypoxanthine guanine phosphoribosyltransferase (HPRT) regulates multiple developmental and metabolic pathways of murine embryonic stem cell neuronal differentiation. PLoS One. 2013;8(10):e74967. Epub 2013/10/17. doi: 10.1371/journal.pone.0074967. PubMed PMID: 24130677; PubMed Central PMCID: PMCPMC3794013.

54. Corsini NS, Peer AM, Moeseneder P, Roiuk M, Burkard TR, Theussl HC, et al. Coordinated Control of mRNA and rRNA Processing Controls Embryonic Stem Cell Pluripotency and 
Differentiation. Cell stem cell. 2018;22(4):543-58 e12. Epub 2018/04/07. doi:

10.1016/j.stem.2018.03.002. PubMed PMID: 29625069.

55. Noden DM, Francis-West P. The differentiation and morphogenesis of craniofacial muscles. Dev Dyn. 2006;235(5):1194-218. Epub 2006/02/28. doi: 10.1002/dvdy.20697s. PubMed PMID: 16502415.

56. Baranauskas A, Paliksa S, Alzbutas G, Vaitkevicius M, Lubiene J, Letukiene V, et al. Generation and characterization of new highly thermostable and processive M-MuLV reverse transcriptase variants. Protein Engineering Design and Selection. 2012;25(10):657-68. doi: 10.1093/protein/gzs034.

57. Piovesan A, Caracausi M, Antonaros F, Pelleri MC, Vitale L. GeneBase 1.1: a tool to summarize data from NCBI gene datasets and its application to an update of human gene statistics. Database (Oxford). 2016;2016. Epub 2016/12/28. doi: 10.1093/database/baw153. PubMed PMID: 28025344; PubMed Central PMCID: PMCPMC5199132.

58. Bian Z, Yu Y, Quan C, Guan R, Jin Y, Wu J, et al. RPL13A as a reference gene for normalizing mRNA transcription of ovarian cancer cells with paclitaxel and 10-hydroxycamptothecin treatments. Molecular medicine reports. 2015;11(4):3188-94. Epub 2014/12/20. doi: 10.3892/mmr.2014.3108. PubMed PMID: 25523336.

59. Gentile AM, Lhamyani S, Coin-Araguez L, Oliva-Olivera W, Zayed H, Vega-Rioja A, et al. RPL13A and EEF1A1 Are Suitable Reference Genes for qPCR during Adipocyte Differentiation of Vascular Stromal Cells from Patients with Different BMI and HOMA-IR. PLoS One.

2016;11(6):e0157002. Epub 2016/06/16. doi: 10.1371/journal.pone.0157002. PubMed PMID: 27304673; PubMed Central PMCID: PMCPMC4909211.

60. Curtis KM, Gomez LA, Rios C, Garbayo E, Raval AP, Perez-Pinzon MA, et al. EF1alpha and RPL13a represent normalization genes suitable for RT-qPCR analysis of bone marrow derived mesenchymal stem cells. BMC molecular biology. 2010;11:61. Epub 2010/08/19. doi: 10.1186/14712199-11-61. PubMed PMID: 20716364; PubMed Central PMCID: PMCPMC2931506.

\section{Figure Legends}

Fig 1: developmental timeline of mouse embryos used in this study

Progress from E11.5 to E18.5 involves marked increases in size and mass and progressive accumulation of somite pairs. Within the head, multiple defined brain structures emerge over this period, and neurogenesis peaks in different brain regions (as indicated). The eye matures, as do teeth. Within the forelimbs, development proceeds from primitive limb bud through to joint and digit formation, maturation and ossification of long bones and emergence of nail primordia. Within the body, the source of haematopoiesis shifts from the yolk sac to the liver, and thence transiently to the spleen before establishing in the bone marrow; the lungs mature through primitive pseudoglandular stages to saccular morphology; the mesonephros of the kidney degenerates and is replaced with the final metanephros. RPE: retinal pigmented epithelium

Fig 2: Raw Cq values and validation 
Box and whisker plots of sample Cq values for each candidate gene used in this study: points represent individual sample Cq values. (A) Initial raw $\mathrm{Cq}$ values revealed a marked variance in $18 \mathrm{~S}$ expression (but not other genes), restricted to samples from a single cDNA synthesis batch (open circles), compared to samples from other cDNA synthesis batches (black points), indicative of impaired random priming. (B) Preparation of fresh cDNA for these samples corrected this discrepancy (filled circles), producing values consistent with other batches (black points). (C) qPCR for $18 \mathrm{~S}$ and the dystrophin isoform dp71 using the initial poor samples (1), the other samples (2) and the fresh preparation (3) shows that impaired random priming prevents accurate quantification of long transcripts.

\section{Fig 3: GeNorm rankings}

Rankings and scores of the fifteen candidate genes assessed by the geNorm algorithm, using the entire dataset (A), whole embryo samples only (B), heads only $(C)$ or forelimbs only (D). High M values represent less stable genes. Genes with $M$ values $<0.5$ (dashed line) are considered acceptable references.

Fig 4: deltaCt rankings

Rankings and scores of the fifteen candidate genes assessed by the deltaCt method, using the entire dataset $(A)$, whole embryo samples only $(B)$, heads only $(C)$ or forelimbs only (D). High dCt scores represent less stable genes.

Fig 5: Bestkeeper rankings

Rankings and scores of the fifteen candidate genes assessed by the Bestkeeper method, using the entire dataset (A), whole embryo samples only (B), heads only $(C)$ or forelimbs only (D). Genes are ranked by their individual Pearson correlation coefficient ( $r$ ) to the bestkeeper derived from all candidate genes: low $r$ values correspond to less stable genes. Dashed line: r value of zero.

Fig 6: Normfinder rankings (ungrouped)

Ungrouped rankings and scores of the fifteen candidate genes assessed by the Normfinder algorithm, using the entire dataset (A), whole embryo samples only $(B)$, heads only $(C)$ or forelimbs only (D). High stability values correspond to less stable genes.

Fig 7: Normfinder rankings (grouped)

Grouped rankings and scores of the fifteen candidate genes assessed by the Normfinder algorithm, using the entire dataset grouped by tissue $(A)$ or by age $(B)$, or whole embryo samples only grouped by age (C), heads grouped by age (D) or forelimbs grouped by age (E). High stability values correspond to less stable genes. The best pair of genes (which may not be the highest scoring individually) and the stability of that pair are indicated (boxes). 
Fig 8: Integrated rankings of the four analysis methods

Geometric mean scores of all fifteen candidate genes from all four algorithms (see methods), assessed as either a complete dataset $(A)$, whole embryos only $(B)$, heads $(C)$ or forelimbs (D). High integrated stability values correspond to less stable genes.

Fig 9: Normalisation reveals HPRT1 and B2M are developmentally upregulated

Mean raw $R Q$ values for HPRT1 (A) and B2M (C) suggest modest expression at earlier stages with marked increases at E18.5. Normalisation with AP3D1, RPL13A and PAK1IP1 $(B$ and $D)$ lowers total coefficient of variation ( $\mathrm{CoV}$ ) and shows both genes are upregulated with increasing gestational age in whole embryos, heads and forelimbs (as indicated). Total CoV values were obtained by summing the individual CoVs per time-point (see methods). Points represent individual $R Q$ values (arbitrary units).

Fig 10: Normalisation reveals CDC40 and HTATSF1 are developmentally downregulated

Mean raw RQ values for CDC40 (A) and HTATSF1 (C) suggest closely-matched but variable expression. Normalisation with AP3D1, RPL13A and PAK1IP1 (B and D) lowers total coefficient of variation ( $\mathrm{CoV}$ ) and shows both genes are modestly downregulated with increasing gestational age in whole embryos, and more markedly downregulated in heads and forelimbs (as indicated). Total CoV values were obtained by summing the individual CoVs per time-point (see methods). Points represent individual $R Q$ values (arbitrary units). 


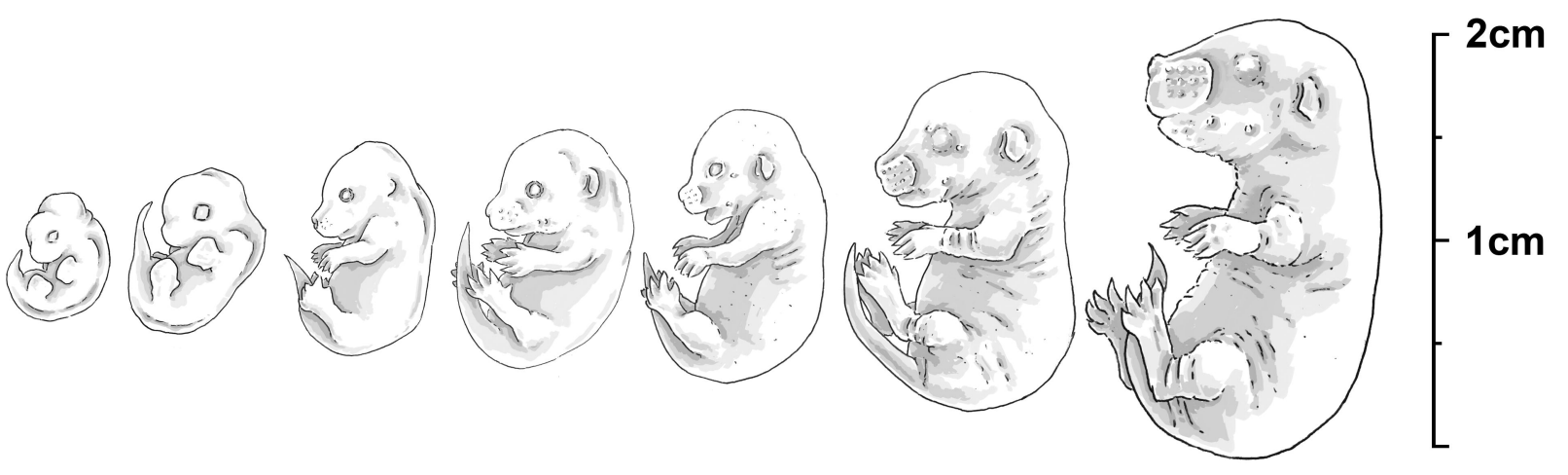

\begin{tabular}{|clc|}
\hline E11.5 & E12.5 & E13.5 \\
\hline $45-47$ & $48-51$ & $52-55$ \\
\hline $\begin{array}{c}\text { 5-vesicle } \\
\text { stage }\end{array}$ & $\begin{array}{c}\text { Cerebellar } \\
\text { Primordium }\end{array}$ & $\begin{array}{c}\text { Choroid } \\
\text { Plexus }\end{array}$ \\
\hline
\end{tabular}

\begin{abstract}
E14.5
\end{abstract}
E15.5

E16.5

E18.5

Gest. Age

$>60$

$>60$

$>60$

Somite pairs

56-60

Corpus

Rostral

Commisure

Callosum Gyrus visible

Brain

Hippocampus

Neurogenesis

Hypothalamus

\title{
Thalamus
}

Cerebral Cortex

\section{Cerebellum}

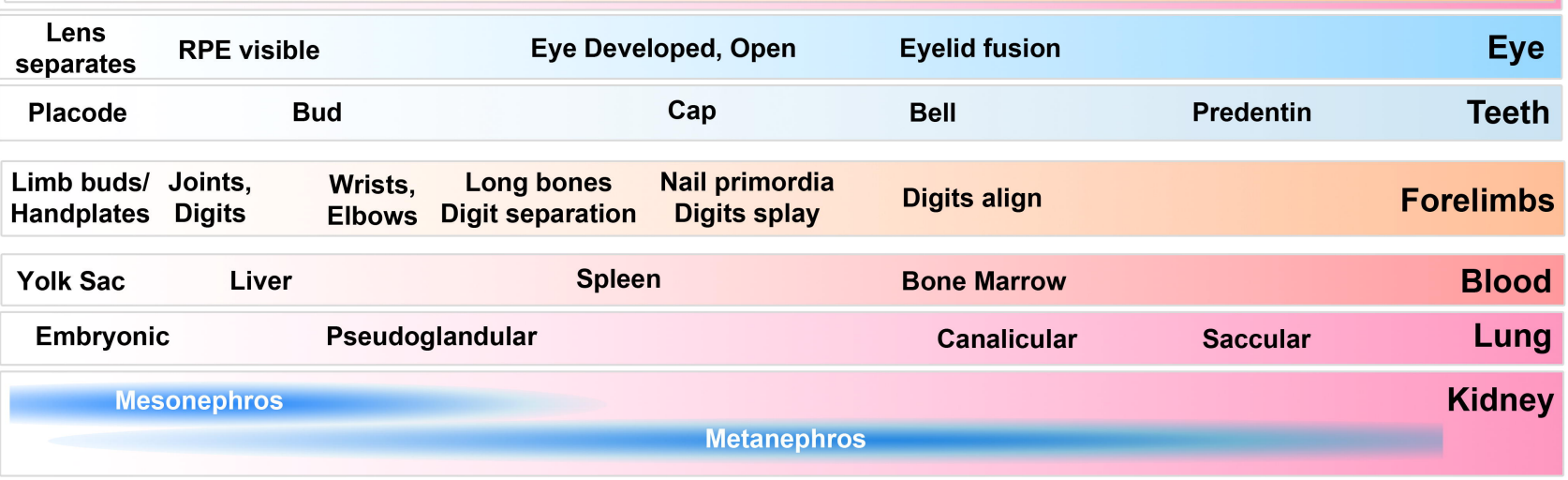




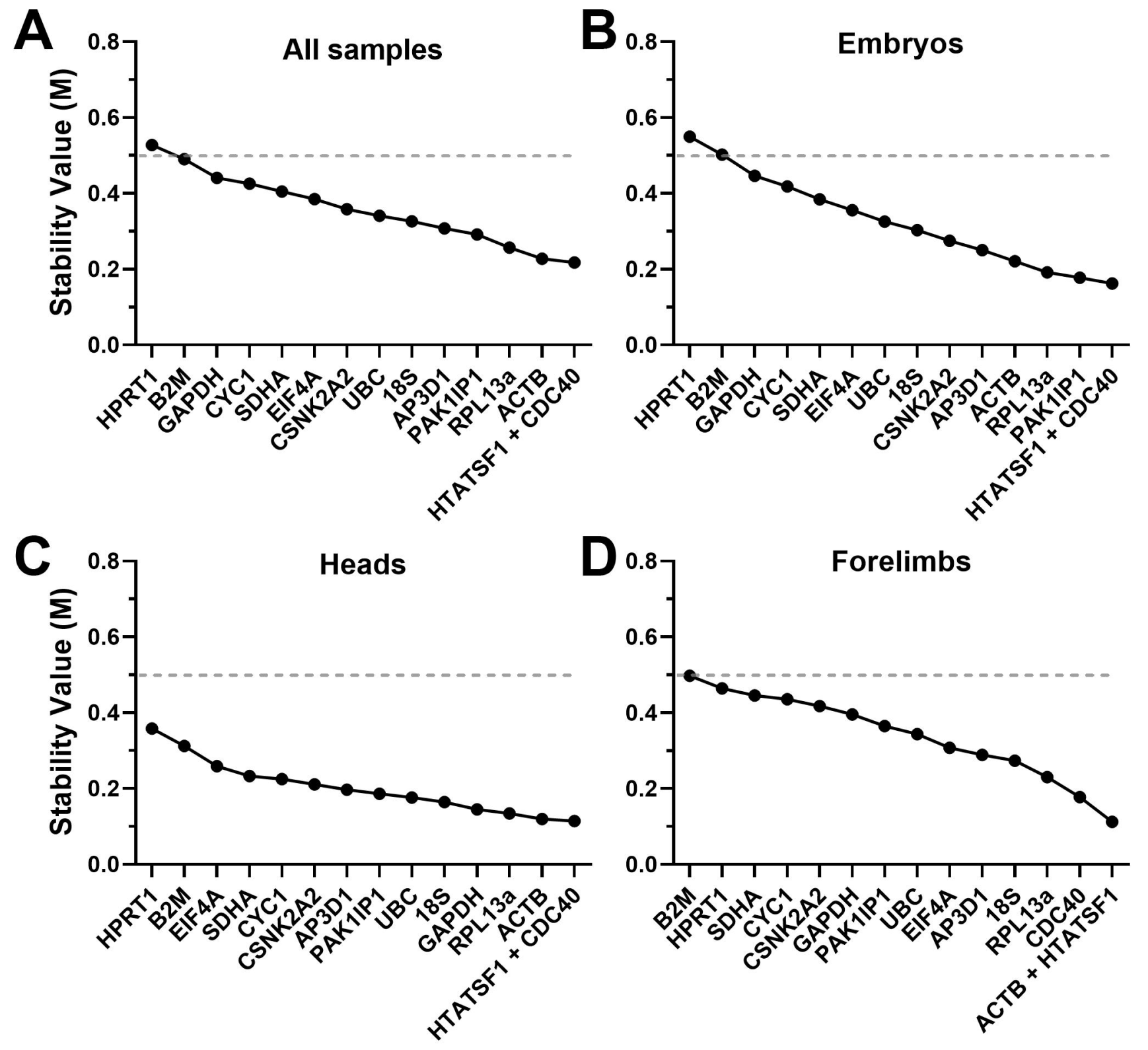



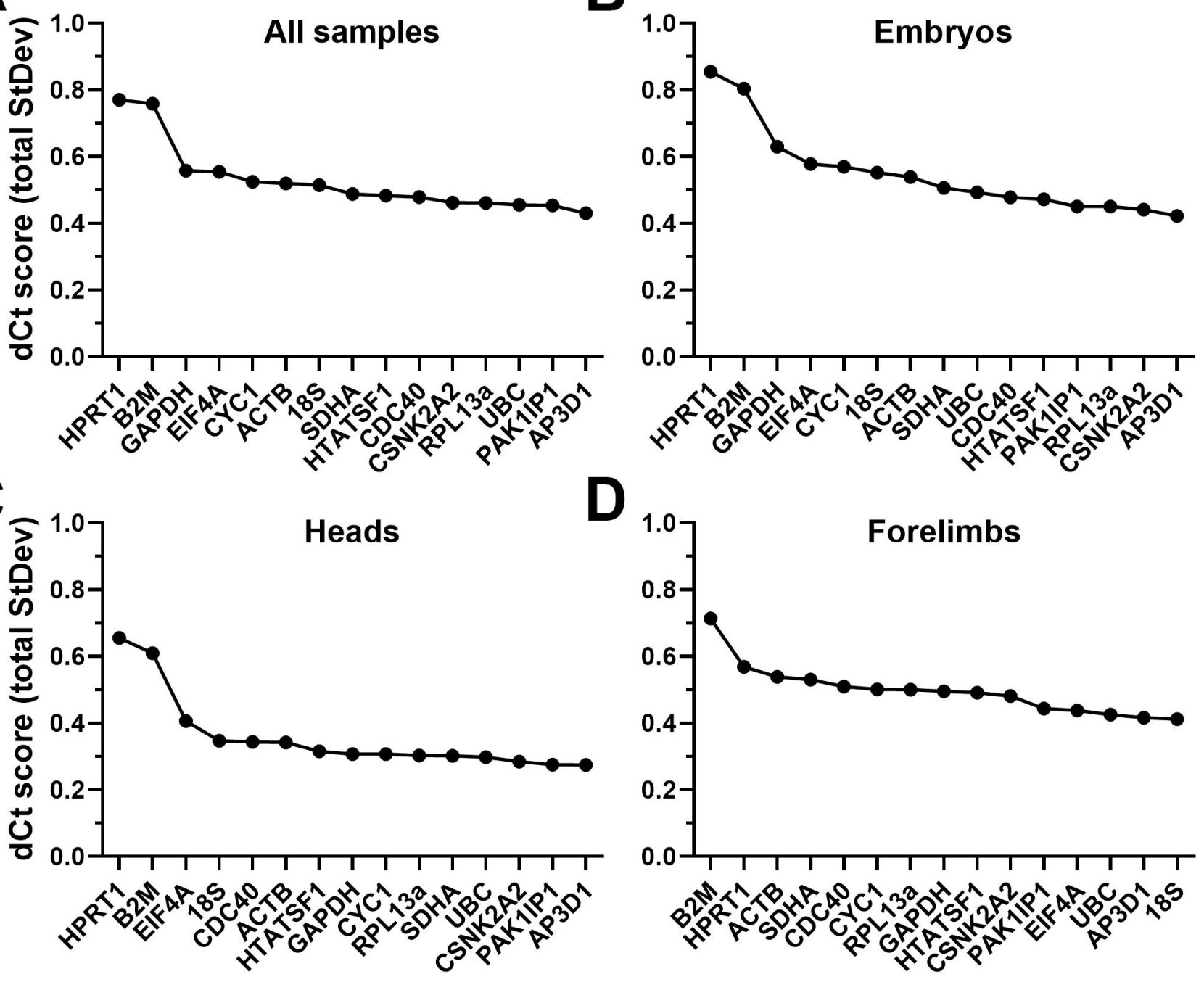
$A$
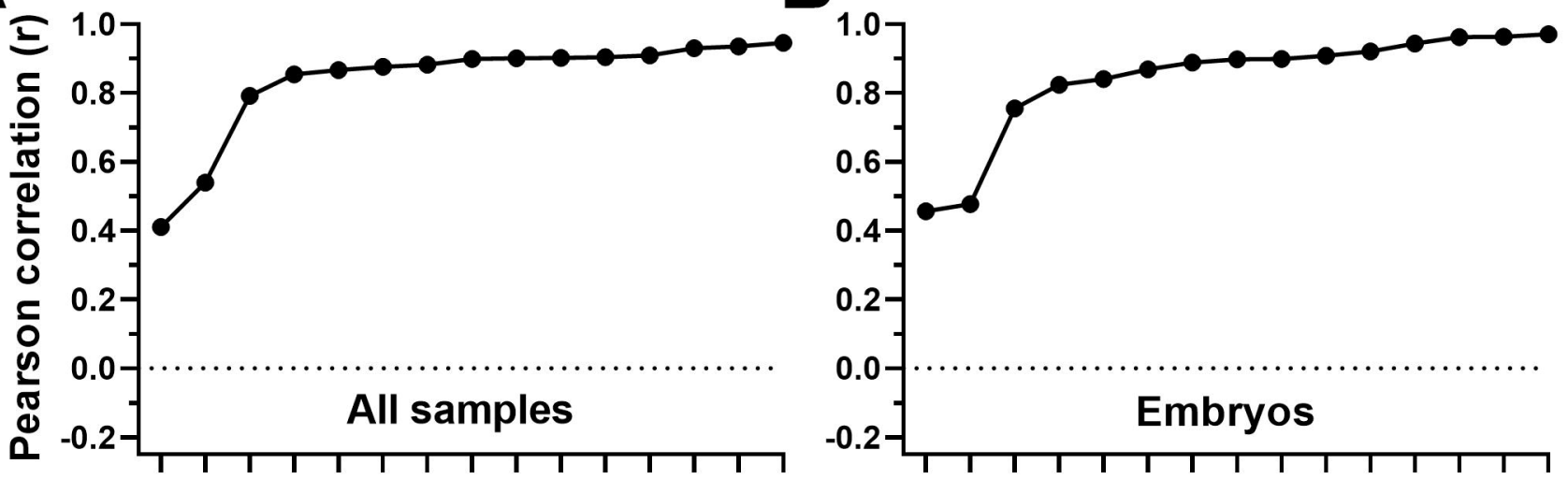

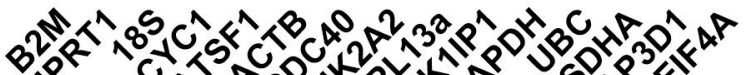

C
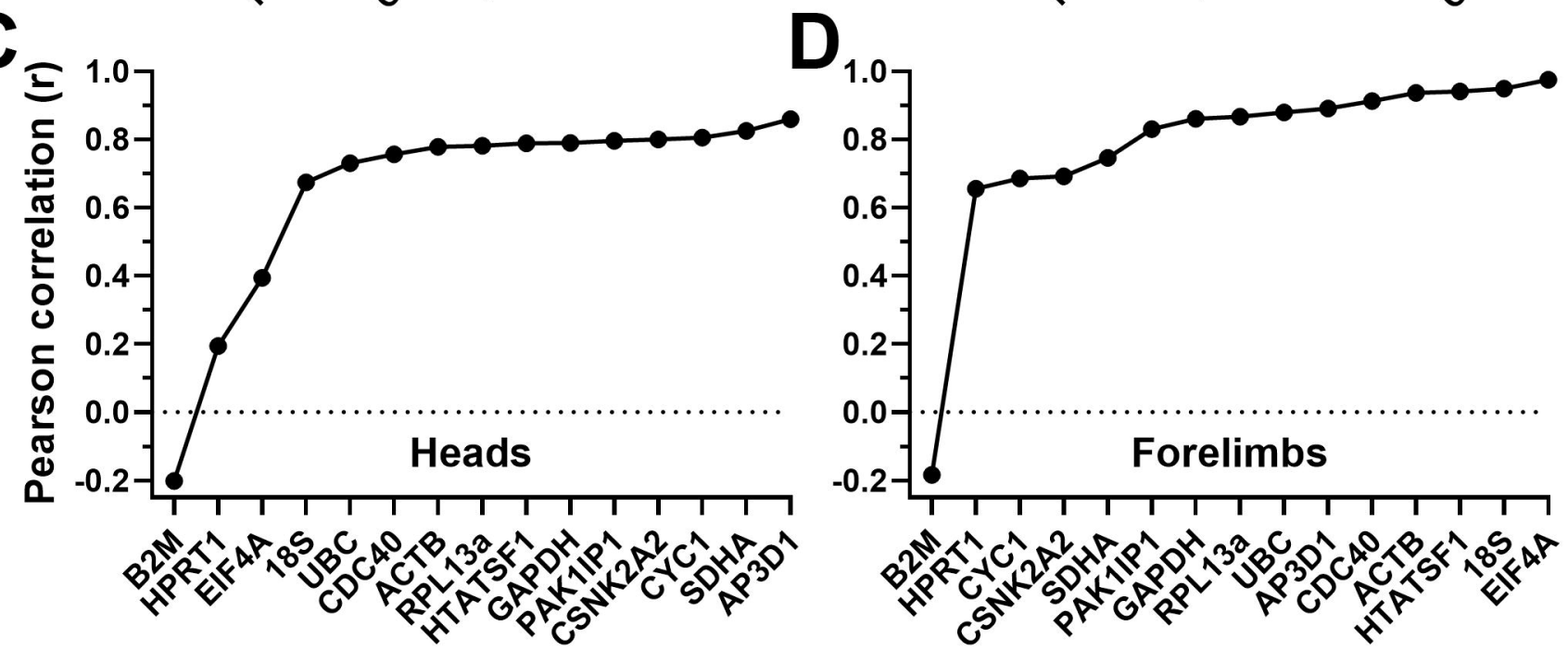
A

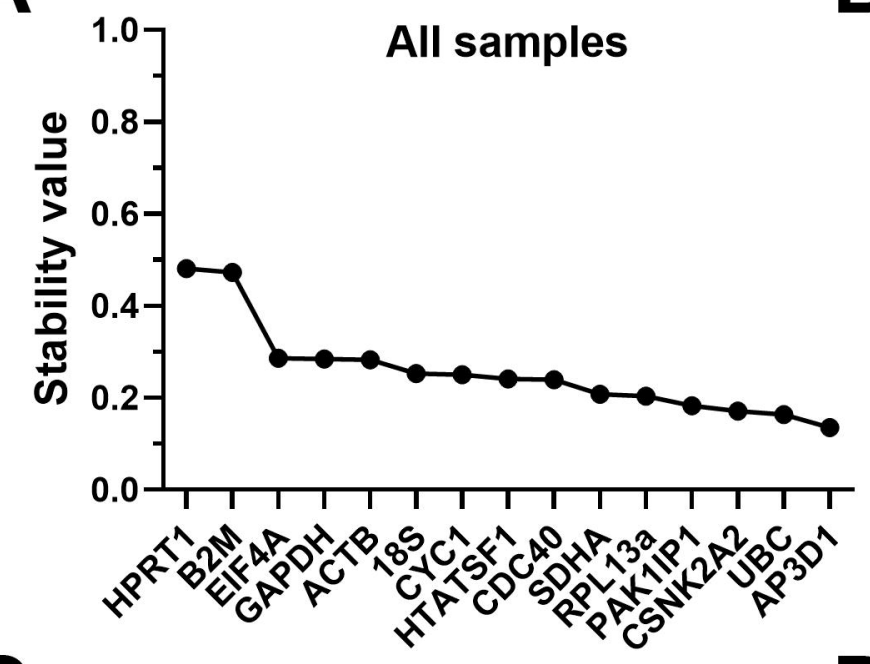

C

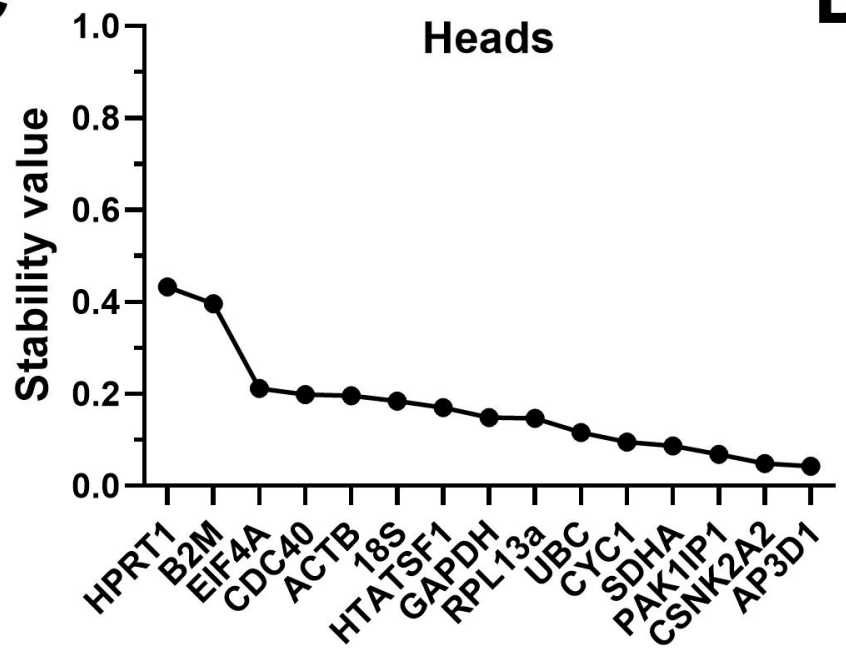

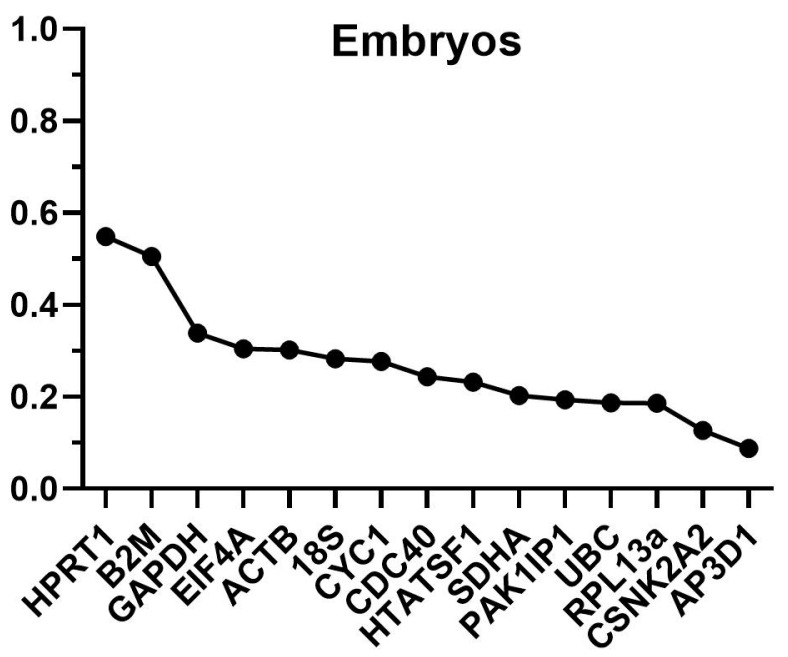

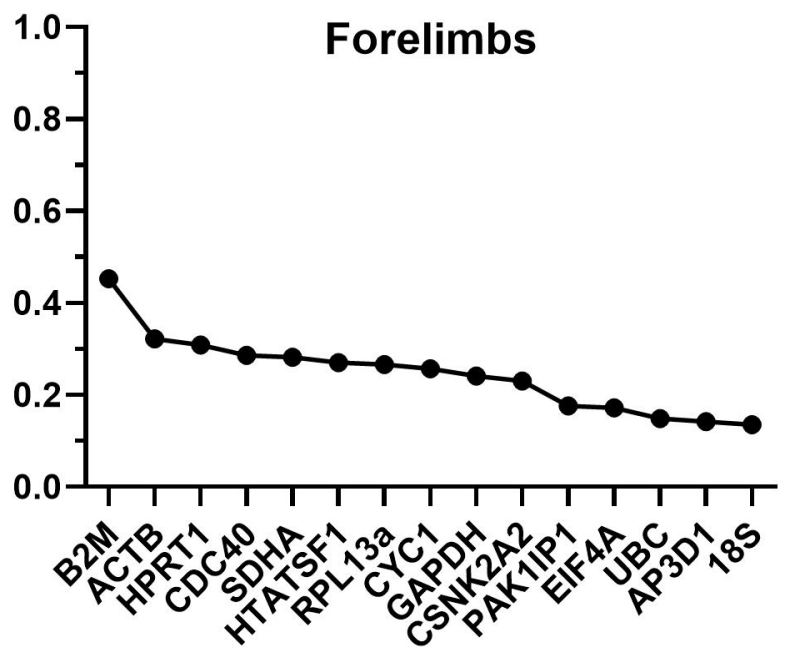



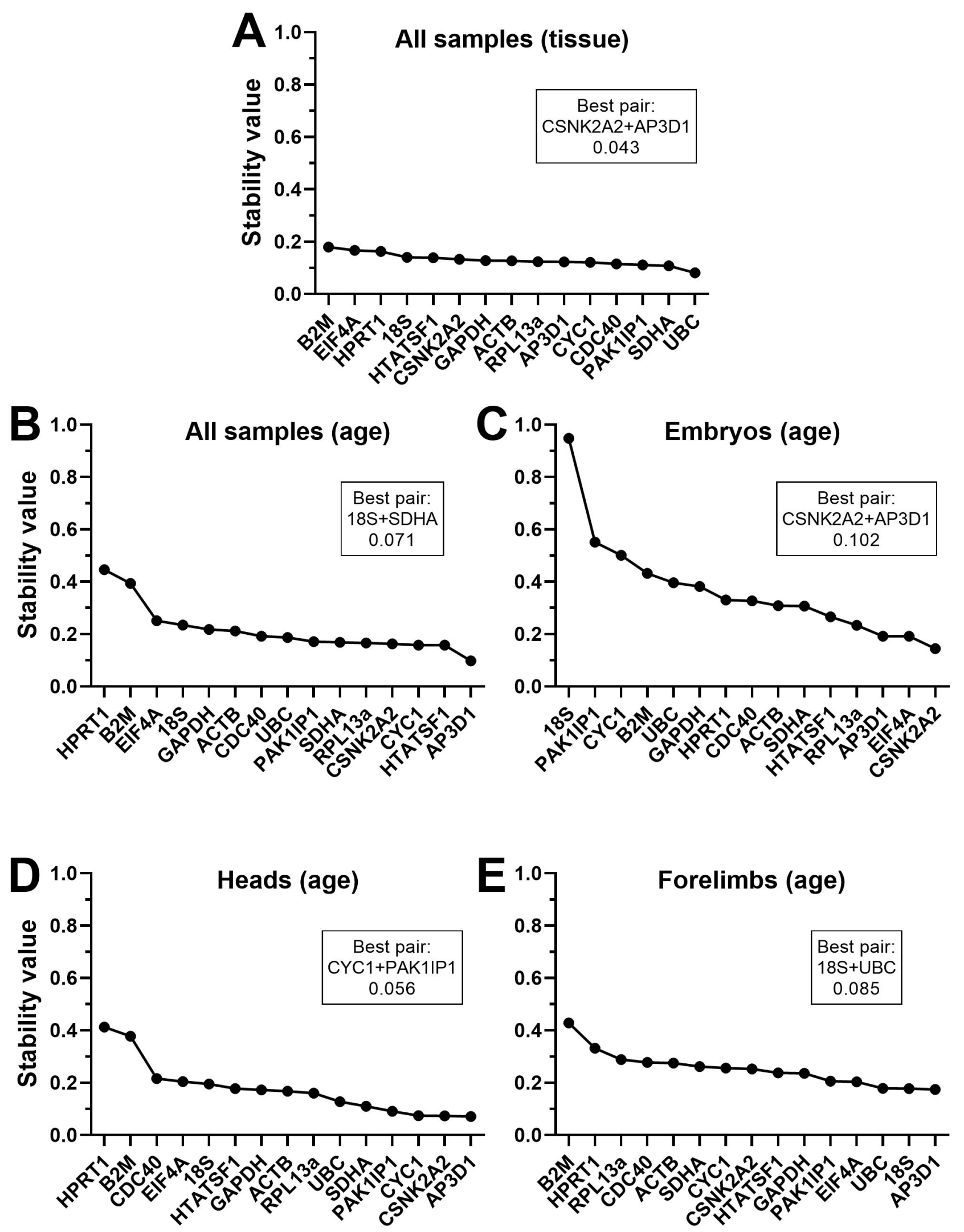

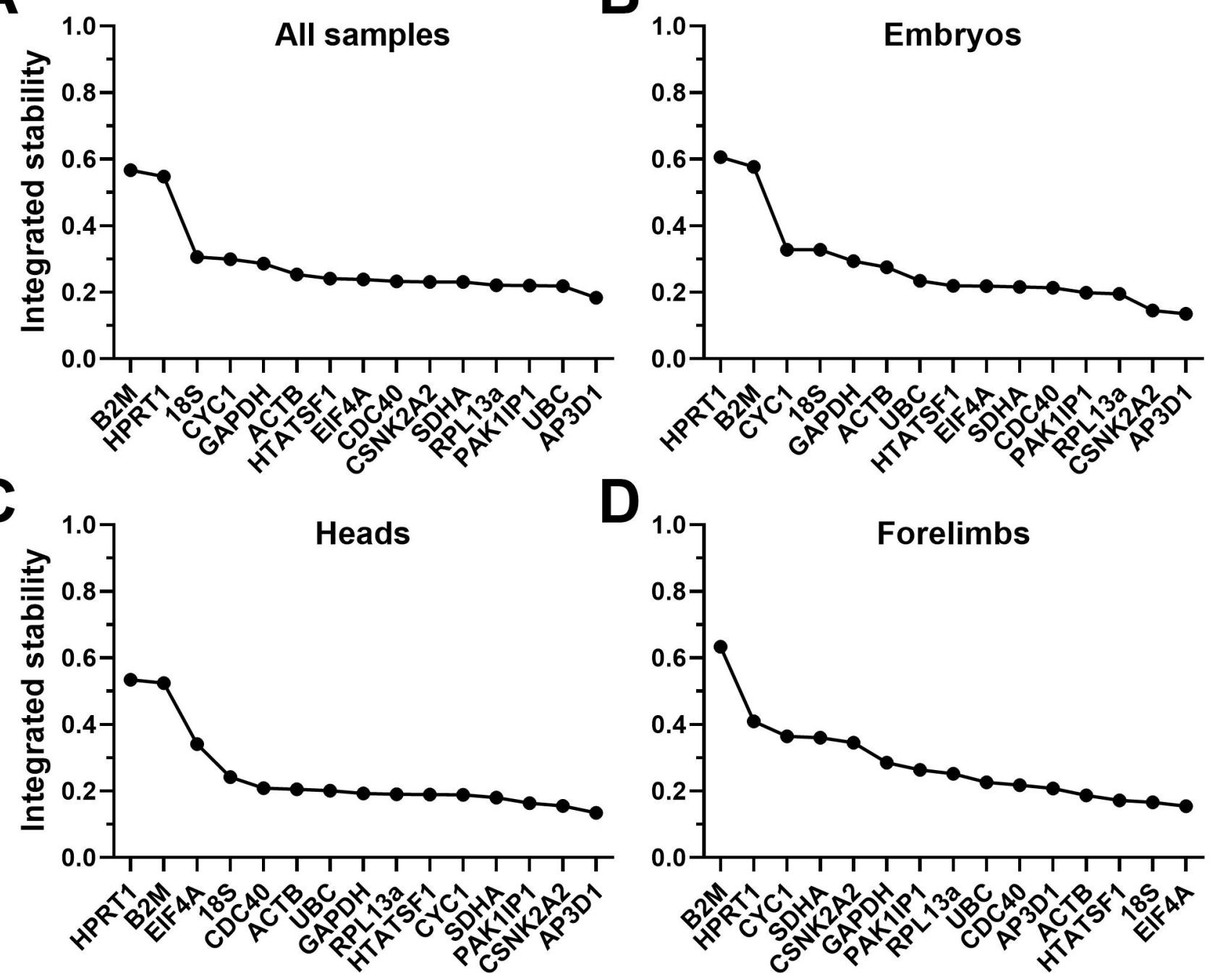
HPRT1 (raw)

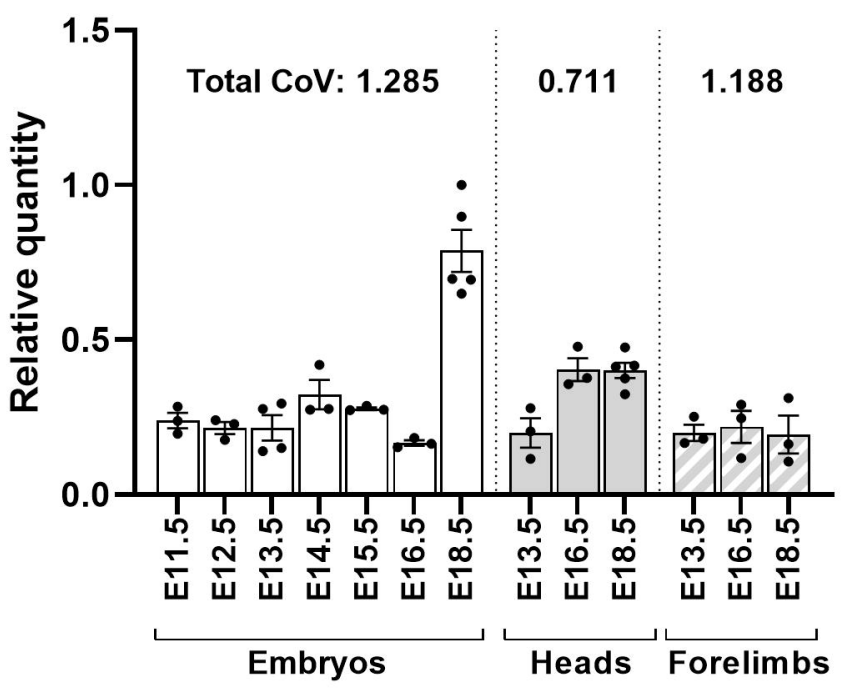

HPRT1 (normalised)

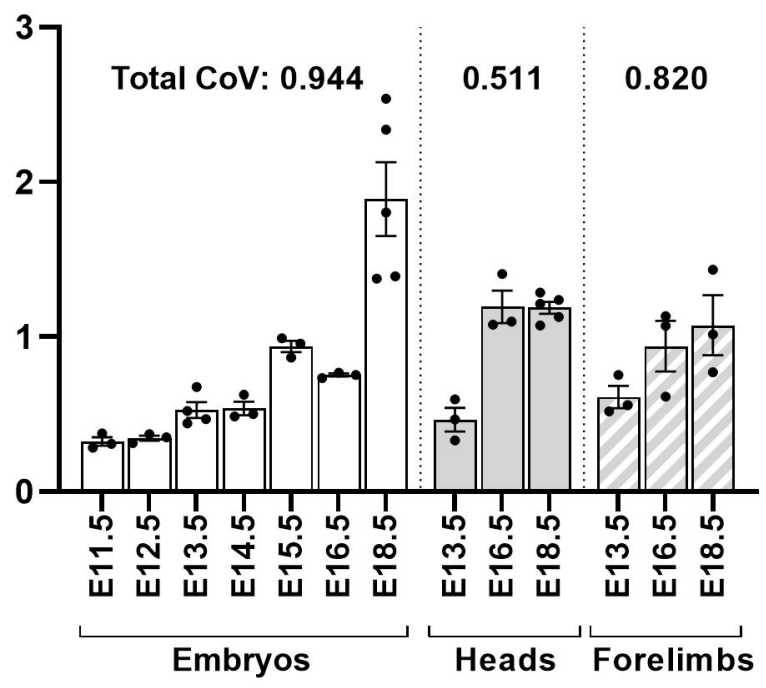

B2M (raw)

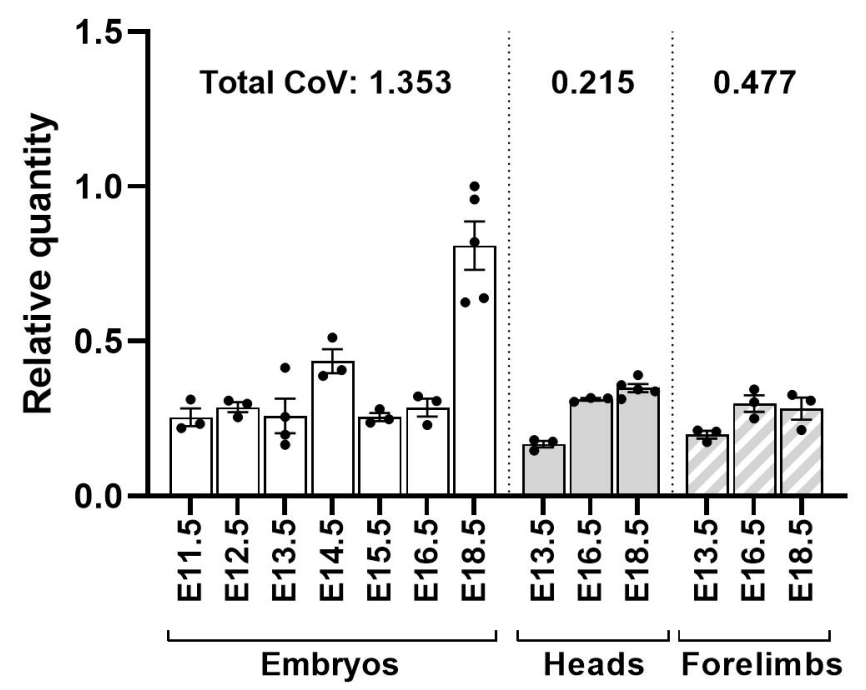

B2M (normalised)

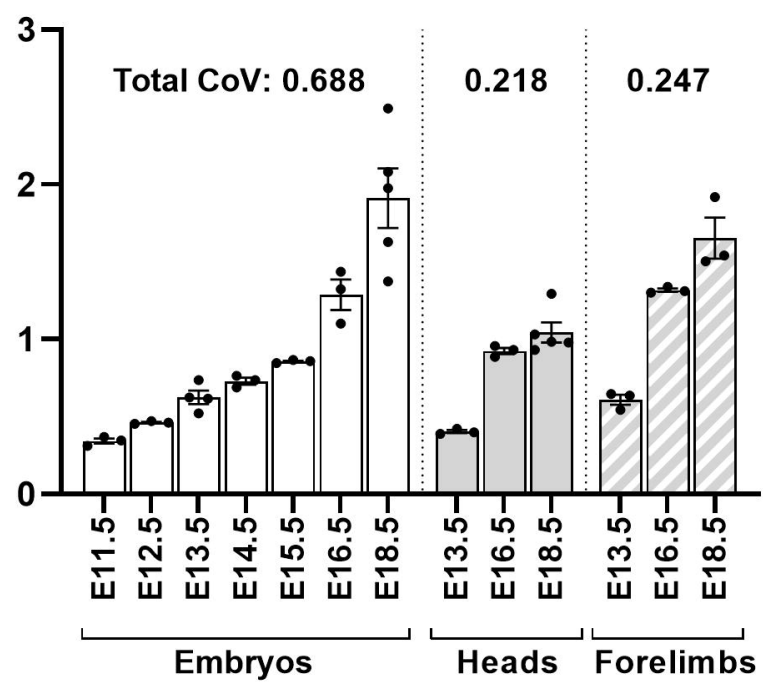



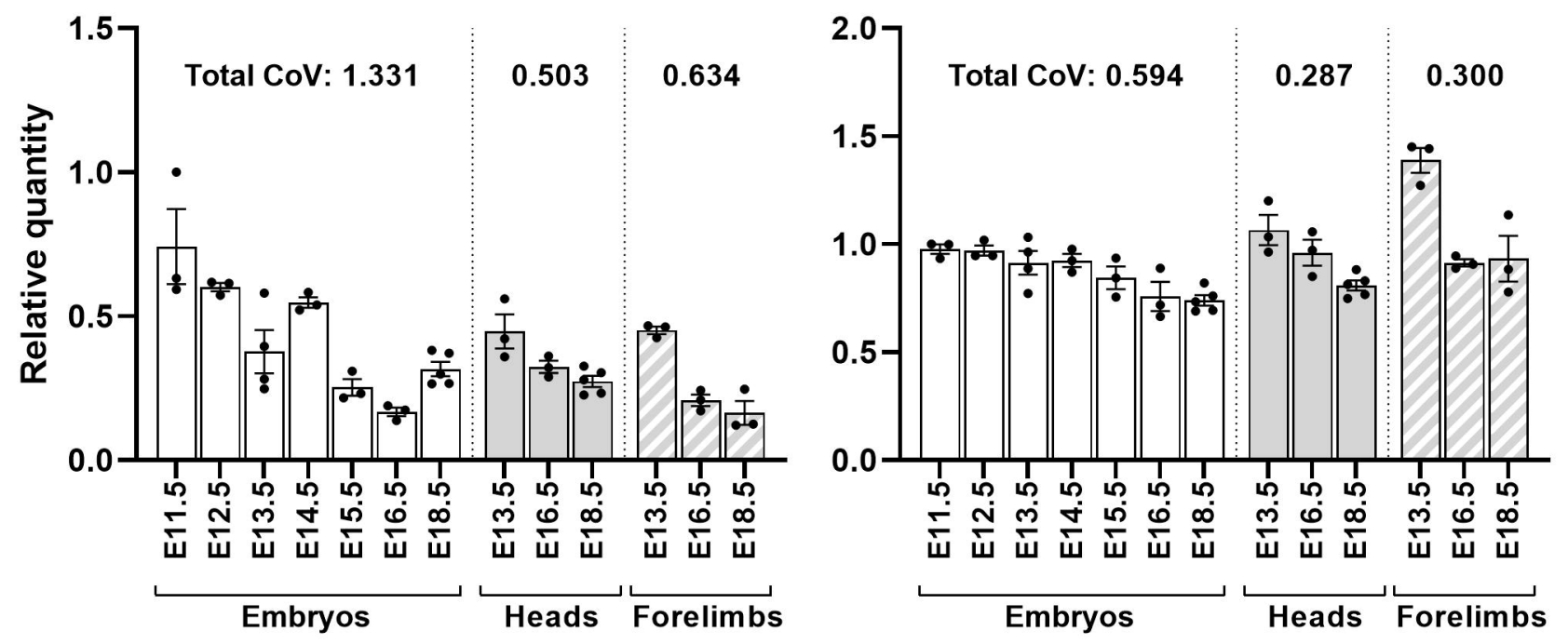

HTATSF1 (raw)

HTATSF1 (normalised)
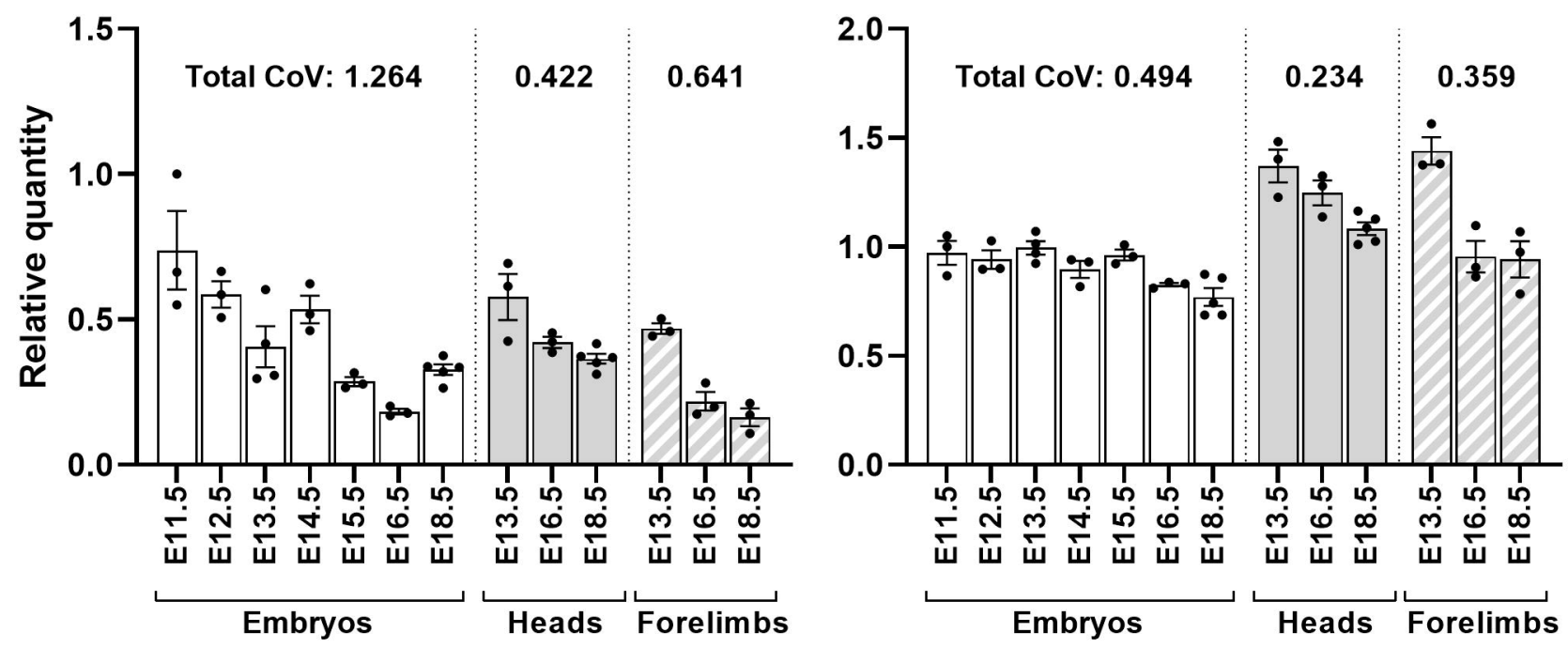